\title{
MOTIVIC STABLE HOMOTOPY GROUPS
}

\author{
DANIEL C. ISAKSEN AND PAUL ARNE ØSTVÆR
}

\begin{abstract}
We survey computations of stable motivic homotopy groups over various fields. The main tools are the motivic Adams spectral sequence, the motivic Adams-Novikov spectral sequence, and the effective slice spectral sequence. We state some projects for future study.
\end{abstract}

\section{INTRODUCTION}

Motivic homotopy theory was developed by Morel and Voevodsky Mor99b MV99] in the 1990's. The original motivation for the theory was to import homotopical techniques into algebraic geometry. For example, it allowed for the powerful theory of Steenrod operations in algebro-geometric cohomology theories. Motivic homotopy theory was essential to the solution of several long-standing problems in algebraic $K$-theory, such as the the Milnor conjecture Voe03b] and the Bloch-Kato conjecture [Voe11.

The past twenty years have witnessed a great expansion of motivic homotopy theory. Just as in classical homotopy theory, the motivic version of stable homotopy groups are among the most fundamental invariants. The goal of this article is to describe what is known about motivic stable homotopy groups and to suggest directions for further study. This topic is just one of several active directions within motivic homotopy theory.

There are at least two motivations for studying motivic stable homotopy groups closely. First, these groups ought to carry interesting arithmetic information about the structure of the ground field; this is essentially the same as the original purpose of motivic homotopy theory.

But another motivation has arisen in recent years. It turns out that the richer structure of motivic stable homotopy theory sheds new light on the structure of the classical stable homotopy groups. In other words, motivic stable homotopy theory is of interest in classical homotopy theory, independently of the applications to algebraic geometry.

The motivic stable homotopy category $\mathbf{S H}(k)$ is constructed roughly as follows. Start with a ground field $k$, and consider the category of smooth schemes over $k$. Expand this category to a larger category with better formal properties. Finally, impose homotopical relations, especially that the affine line $\mathbb{A}^{1}$ is contractible. The result of this process is the unstable motivic homotopy category over $k$.

2010 Mathematics Subject Classification. Primary 14F42, 55T15, 55Q45 .

Key words and phrases. stable motivic homotopy group, stable motivic homotopy theory, Adams spectral sequence, Adams-Novikov spectral sequence, effective spectral sequence.

The author was supported by NSF grant DMS-1202213.

The author was supported by the RCN Frontier Research Group Project no. 250399 "Motivic Hopf equations", a Friedrich Wilhelm Bessel Research Award from the Alexander von Humboldt Foundation, and a Nelder Visiting Fellowship from Imperial College London. 
Next, identify a bigraded family $S^{p, q}$ of unstable spheres for $p \geq q \geq 0$. These spheres are smash products of the topological circle $S^{1,0}$ and the algebraic circle $S^{1,1}=\mathbb{A}^{1}-0$. Then stabilize with respect to this bigraded family to obtain motivic stable homotopy theory.

A key property of the classical stable homotopy category is that every spectrum is (up to homotopy) built from spheres by homotopy colimits. The motivic analogue of this property does not hold. In other words, there exist motivic spectra that are not cellular. Non-trivial field extensions of the base field are examples of noncellular motivic spectra. Elliptic curves are another source of such examples. In both cases, interesting arithmetic properties of the algebraic object interfere.

One important consequence is that the motivic stable homotopy groups $\pi_{p, q} X=$ $\left[S^{p, q}, X\right]$ do not detect equivalences. One solution to this problem is to consider motivic stable homotopy sheaves. The idea is to keep track of maps not only from spheres, but maps out of all smooth schemes. By construction of the motivic stable homotopy category $\mathbf{S H}(k)$, these smooth schemes serve as generators for the category. Therefore, motivic stable homotopy sheaves do detect motivic equivalences.

We will not pursue the motivic stable homotopy sheaf perspective further in this article. Instead, we will focus just on the groups $\pi_{p, q}=\left[S^{p, q}, S^{0,0}\right]$. The precise relationship is that the motivic stable homotopy groups are the global sections of the motivic stable homotopy sheaves. For cellular motivic spectra, the motivic stable homotopy groups do detect equivalences, and the most commonly studied motivic spectra are typically cellular. Moreover, the stable homotopy sheaves are "unramified", which means that they are actually determined by their sections over fields. So a thorough understanding of motivic stable homotopy groups over arbitrary fields leads back to complete information about the sheaves as well.

1.1. Completions. Classically, Serre's finiteness theorem says that in positive dimensions, every stable homotopy group is finite. Therefore, it is enough to compute the $p$-completions of the stable homotopy groups for all primes $p$. Tools such as the Adams spectral sequence and the Adams-Novikov spectral sequence allow for the calculation of these $p$-completions.

As one would expect, the motivic situation is more involved. The motivic stable homotopy groups are not finite in general. For example, the group $\pi_{-1,-1}$ contains a copy of the multiplicative group $k^{\times}$of the base field. When $k=\mathbb{C}$, this is an uncountable group that is infinitely divisible. Therefore, all of the $p$-completions of $\pi_{-1,-1}$ vanish, even though $\pi_{-1,-1}$ itself is non-zero. Nevertheless, we will compute $p$-completions, even though there is a certain loss of information.

A full accounting of the machinery of completion in the motivic context is beyond the scope of this article. We refer to [HKO11a and DI10. The completions arise naturally when considering convergence for the Adams and Adams-Novikov spectral sequences.

The situation with completions is in fact even more complicated. The first motivic Hopf map $\eta$ is the projection $\mathbb{A}^{2}-0 \rightarrow \mathbb{P}^{1}$, i.e., a map $S^{3,2} \rightarrow S^{2,1}$ representing an element in the motivic stable homotopy group $\pi_{1,1}$. We need to complete with respect to $\eta$ in order to obtain convergence for the relevant spectral sequences. The 
arithmetic square

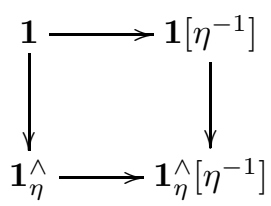

is a homotopy pullback for the motivic sphere spectrum 1, as discussed in RSØa, Lemma 3.9]. Thus, information about the $\eta$-completion and the $\eta$-localization (and how they fit together) leads to information about the uncompleted motivic stable homotopy groups.

Because of our computational perspective, we will mostly be interested in torsion in the motivic stable homotopy groups. The articles [Mor06] and ALP17] study the rationalized motivic stable homotopy groups. After inverting 2 , the motivic sphere spectrum splits into two summands, and the rationalizations of these summands can be described in terms of motivic cohomology with rational coefficients. We will not pursue this perspective further in this article.

1.2. Organization. In Section 2, we introduce the motivic Adams spectral sequence, which is one of the key tools for computing stable motivic homotopy groups. The discussion includes background on Milnor $K$-theory of fields, the motivic cohomology of a point, the structure of the motivic Steenrod algebra, and Ext groups over the motivic Steenrod algebra.

In Section 3, we introduce the motivic effective slice spectral sequence, which is the other key tool for computing stable motivic homotopy groups. We explain how the effective slice filtration arises in the stable motivic homotopy category $\mathbf{S H}(k)$, how to compute the layers of this filtration for the motivic sphere spectrum, and how to compute the layers of a few other related motivic spectra.

In Sections 4 and 5, we specialize to the base fields $\mathbb{C}$ and $\mathbb{R}$. We describe results derived from the motivic Adams spectral sequence. This includes calculations of the $\eta$-periodic motivic stable homotopy groups, significant implications for the calculation of classical stable homotopy groups, and exotic nilpotence and periodicity properties.

In Section 6, we discuss calculations over other fields. This includes computations over finite fields, a general discussion of graded commutativity, Milnor-Witt $K$ theory and its relationship to the groups $\pi_{n, n}$, and some results on the groups $\pi_{n+1, n}$.

Section 7 covers calculations of stable homotopy groups of other motivic spectra, including the $K$-theory spectra KGL, kgl, KQ, and kq; motivic (truncated) Brown-Peterson spectra $\mathbf{M B P}\langle n\rangle$; and the Witt theory spectrum $\mathbf{K W}$. The key tool here is the motivic effective slice spectral sequence. We also describe how computations involving $\mathbf{K W}$ are related to Milnor's conjecture on quadratic forms.

Finally, Section 8 provides some open-ended projects that may motivate further work in the subject of stable motivic homotopy groups.

1.3. Notation. Our convention for motivic grading is to use the notation $(i, j)$, where $i$ is the topological dimension and $j$ is the motivic weight. Some authors use the notation $(i-j)+j \alpha$, which illuminates the analogy to equivariant homotopy theory. 
The following table of notation is provided for the reader's convenience. Detailed descriptions are provided throughout the manuscript.

- $K_{*}^{M}(k)$ is the Milnor $K$-theory of the ground field $k$. (Section 2.1)

- $\mathbb{M}_{p}=H^{*, *}\left(k ; \mathbb{F}_{p}\right)$ is the motivic cohomology of the ground field $k$ with $\mathbb{F}_{p}$ coefficients. (Theorem 2.7)

- $A_{*}=A_{*}^{k}$ is the dual Steenrod algebra of motivic cohomology operations. The prime is implicit in this notation. (Section 2.2)

- $\operatorname{Ext}_{k}=\operatorname{Ext}_{A}\left(\mathbb{M}_{p}, \mathbb{M}_{p}\right)$ is the cohomology of the motivic Steenrod algebra, which serves as the $E_{2}$-page of the motivic Adams spectral sequence that converges to the completed motivic stable homotopy groups. (Section 2.3)

- $\pi_{i, j}=\pi_{i, j}^{k}$ is the motivic stable homotopy group over $k$ in degree $(i, j)$, completed at some prime that is implicit and also at $\eta$.

- $\Pi_{m}^{k}=\bigoplus_{n \in \mathbb{Z}} \pi_{m+n, n}^{k}$ is the $m$ th Milnor-Witt stem. Sometimes it is more convenient to study all of the groups in $\Pi_{m}^{k}$ at once, rather than one at a time. (Sections 5.1 and 6.3)

- $K_{*}^{M W}(k)$ is the Milnor-Witt $K$-theory of a field $k$. (Section 6.2)

- $\mathrm{f}_{q}(\mathbf{E})$ is the $q$ th effective slice cover of a motivic spectrum E. (Section 3.1)

- $\mathrm{s}_{q}(\mathbf{E})$ is the $q$ th layer of a motivic spectrum $\mathbf{E}$ with respect to the effective slice filtration, i.e., the $q$ th slice of $\mathbf{E}$. (Section 3.1)

- $\tilde{\mathrm{f}}_{q}(\mathbf{E})$ is the $q$ th very effective slice cover of a motivic spectrum $\mathbf{E}$. (Section 3.1)

- $\mathbf{S H}(k)$ is the motivic stable homotopy category over the base field $k$. (Section 3 ).

- $\mathbf{1}=S^{0,0}$ is the motivic sphere spectrum that serves as the unit object of the stable motivic homotopy category $\mathbf{S H}(k)$.

- $S^{i, j}$ is the motivic sphere spectrum of dimension $(i, j)$, and $\Sigma^{i, j}$ represents the suspension functor given by smashing with $S^{i, j}$.

- $\mathbf{M} R$ is the motivic Eilenberg-Mac Lane spectrum that represents motivic cohomology with coefficients in $R$. Usually $R$ is $\mathbb{Z}$ or $\mathbb{Z} / n$. (Sections 3.2 and (7.1)

- KGL is the $(2,1)$-periodic algebraic $K$-theory motivic spectrum. (Sections 3.3 and 7.2 .

- $\mathbf{k g l}=\mathrm{f}_{0} \mathbf{K G L}=\tilde{\mathrm{f}}_{0} \mathbf{K G L}$ is the 0th effective slice cover of $\mathbf{K G L}$, or equivalently the 0th very effective slice cover of KGL. (Sections 3.1 and 7.4)

- $\mathbf{K Q}$ is the $(8,4)$-periodic Hermitian $K$-theory motivic spectrum. (Sections 3.3 and 7.4

- $\mathbf{k q}=\tilde{\mathrm{f}}_{0}(\mathbf{K Q})$ is the 0 th very effective slice cover of $\mathbf{K Q}$. (Sections 3.1 and 7.4)

- $\mathbf{K W}$ is the Witt theory spectrum obtained from $\mathbf{K Q}$ by inverting the first motivic Hopf map $\eta$. (Sections 3.3 and 7.5)

- MGL is the motivic algebraic cobordism spectrum, and MU is the analogous classical complex cobordism spectrum. (Section 3.2)

- MBP is the motivic version of the Brown-Peterson spectrum, and $\mathbf{M B P}\langle n\rangle$ is the truncated version, while $\mathbf{B P}$ and $\mathbf{B P}\langle n\rangle$ are the classical analogues. These constructions depend on the choice of some prime, which is implicit in the notation. (Sections 3.2 and 7.3 ) 
1.4. Acknolwedgements. The authors appreciate constructive feedback from J. Hornbostel, O. Röndigs, M. Spitzweck, and G. Wilson.

\section{The motivic Adams spectral sequence}

Our techniques for computing motivic stable homotopy groups are fundamentally cohomological in nature. So our discussion begins with the computational properties of motivic cohomology.

2.1. Milnor $K$-theory and the cohomology of a point. Let $k$ be a field. The Milnor $K$-theory $K_{*}^{M}(k)$ of $k$ is a graded ring constructed as follows [Mil69]. Let $K_{0}^{M}(k)$ be $\mathbb{Z}$, and let $K_{1}^{M}(k)$ be the abelian group $k^{\times}$. This presents a notational confusion, because the group structure on $k^{\times}$is multiplicative, but we would like to think of $k^{\times}$as an additive group. We use the symbol $[a]$ to represent the element of $K_{1}^{M}(k)$ corresponding to the element $a$ of $k^{\times}$. Then we have identities such as

$$
[a b]=[a]+[b] .
$$

Now $K_{*}^{M}(k)$ is defined to be the graded commutative ring generated by the elements of $K_{1}^{M}(k)$ subject to the Steinberg relations

$$
[a] \cdot[1-a]=0
$$

in $K_{2}^{M}(k)$ for all $a$ in $k-\{0,1\}$.

The Milnor $K$-theory of familiar fields can be wildly complicated. For example, $K_{1}^{M}(\mathbb{C})$ is an uncountable infinitely divisible abelian group. Fortunately, we will usually consider the much simpler Milnor $K$-theory modulo $p$, i.e., $K_{*}^{M}(k) / p$, for a fixed prime $p$.

We give several standard examples of Milnor $K$-theory computations. More details can be found in [Mil69, Wei13, Section III.7], or [Mag02, Chapter 14E].

Example 2.1. $K_{*}^{M}(\mathbb{C}) / p=\mathbb{F}_{p}$, concentrated in degree 0, since every element of $\mathbb{C}^{\times}$is a $p$ th power of some other element.

Example 2.2. $K_{*}^{M}(\mathbb{R}) / 2=\mathbb{F}_{2}[\rho]$, where $\rho$ is another name for the element $[-1]$ in $K_{1}^{M}(\mathbb{R})$. This calculation follows from the observation that every non-negative real number is a square. On the other hand, $K_{*}^{M}(\mathbb{R}) / p=\mathbb{F}_{p}$ if $p$ is odd, since every element of $\mathbb{R}^{\times}$is a $p$ th power of some other element.

In general, let $\rho$ be the element $[-1]$ in $K_{1}^{M}(k) / 2$ for any field $k$. Note that $\rho$ is zero if and only if -1 is a square in $k$. We will see later in Section 2.2 that $\rho$ plays a central role in the structure of the motivic Steenrod algebra.

Example 2.3. If $p \equiv 1 \bmod 4$, then $K_{*}^{M}\left(\mathbb{Q}_{p}\right) / 2=\mathbb{F}_{2}[\pi, u] /\left(\pi^{2}, u^{2}\right)$, where $\pi=[p]$ and $u=[a]$ for an element $a$ in $\mathbb{Q}_{p}^{\times}$that maps to a non-square in $\mathbb{F}_{p}^{\times}$.

Example 2.4. If $p \equiv 3 \bmod 4$, then $K_{*}^{M}\left(\mathbb{Q}_{p}\right) / 2=\mathbb{F}_{2}[\pi, \rho] /\left(\rho^{2}, \rho \pi+\pi^{2}\right)$, where $\pi=[p]$ and $\rho=[-1]$.

Example 2.5. $K_{*}^{M}\left(\mathbb{Q}_{2}\right) / 2=\mathbb{F}_{2}[\pi, \rho, u] /\left(\rho^{3}, u^{2}, \pi^{2}, \rho u, \rho \pi, \rho^{2}+u \pi\right)$, where $\pi=[2]$, $\rho=[-1]$, and $u=[5]$.

Example 2.6. $K_{*}^{M}\left(\mathbb{F}_{q}\right)$ equals $\mathbb{Z}[u] / u^{2}$, where $u=[a]$ for any generator $a$ of $\mathbb{F}_{q}^{\times}$. 


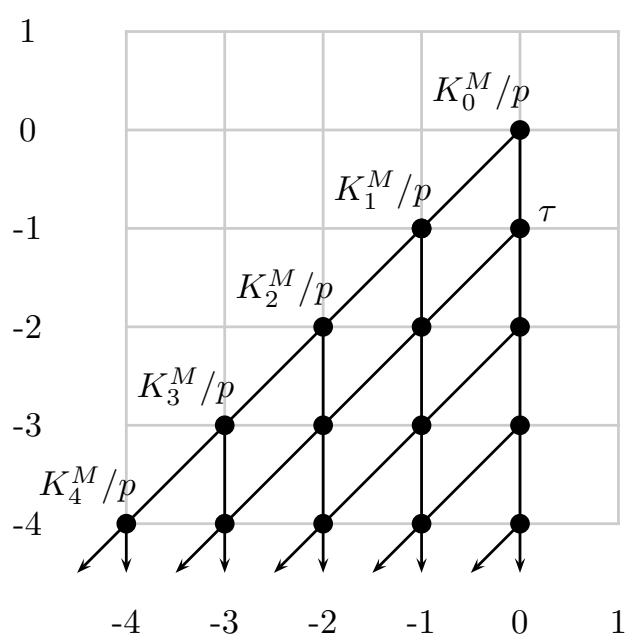

Figure 1. The cohomology of a point. The grading is homological to align with later homotopy group calculations. The topological degree is on the $x$-axis, and the motivic weight is on the $y$-axis. Each dot in column $-n$ represents a copy of $K_{n}^{M}(k) / p$. Vertical lines indicate multiplication by $\tau$. Diagonal lines connect the source and target of multiplications by elements of $K_{1}^{M}(k) / p$.

For us, the point of these Milnor $K$-theory calculations is that we can use them describe the motivic cohomology of a point. Motivic cohomology is bigraded, where the first grading corresponds to the classical topological degree, and the second corresponds to the motivic weight. If $k$ contains a primitive $p$ th root of unity $\zeta_{p}$, we let $\tau$ denote the corresponding generator of $H^{0,1}\left(k ; \mathbb{F}_{p}\right) \cong \mathbb{Z} / p$.

Theorem 2.7. Voe03b Voe11 Suppose that $p$ and char $(k)$ are coprime, and that $k$ contains a primitive pth root of unity. Then the motivic cohomology $\mathbb{M}_{p}=$ $H^{*, *}\left(k ; \mathbb{F}_{p}\right)$ with coefficients in $\mathbb{F}_{p}$ is isomorphic to

$$
\frac{K_{*}^{M}(k)}{p}[\tau]
$$

where $K_{n}^{M}(k) / p$ has degree $(n, n)$, and $\tau$ has degree $(0,1)$.

Figure 1 graphically depicts the calculation in Theorem 2.7.

2.2. The motivic Steenrod algebra. Our next task is to record the structure of the motivic Steenrod algebra [HKØ17], Voe03a]. We continue to assume that the base field $k$ contains a primitive $p$ th root of unity and that $\operatorname{char}(k)$ and $p$ are coprime, so that we rely on the calculation of Theorem 2.7 .

In classical topology, the dual Steenrod algebra is easier to describe than the Steenrod algebra itself. This asymmetry arises from the fact that the coproduct structure (i.e., the Cartan formula) is simpler than the product structure (i.e., the Adem relations).

In the motivic context, the advantages of the dual Steenrod algebra are even more pronounced. The motivic Adem relations possess some non-trivial complications 
that make them difficult to even write down correctly. Beware that more than one published version of the motivic Adem relations has mistakes. See HKØ17, Theorem 5.1] for the correctly formulated relations.

Let $p$ be an odd prime. The dual motivic Steenrod algebra $A_{*}$ is a commutative $\mathbb{M}_{p}$-algebra, where $\mathbb{M}_{p}=H^{*, *}\left(k ; \mathbb{F}_{p}\right)$ is the cohomology of a point (see Theorem 2.7). It is generated by elements $\tau_{i}$ for $i \geq 0$ and $\xi_{j}$ for $j \geq 1$, subject to the relations $\tau_{i}^{2}=0$. Here $\tau_{i}$ has degree $\left(2 p^{i}-1, p^{i}-1\right)$, and $\xi_{j}$ has degree $\left(2 p^{j}-2, p^{j}-1\right)$. The coproduct $\Delta$ on $A_{*}$ is described by

$$
\Delta\left(\tau_{i}\right)=\tau_{i} \otimes 1+\sum_{k=0}^{i} \xi_{i-k}^{p^{k}} \otimes \tau_{k}
$$

and

$$
\Delta\left(\xi_{j}\right)=\sum_{k=0}^{j} \xi_{j-k}^{p^{k}} \otimes \xi_{k} .
$$

In order to properly interpret these formulas, we adopt the usual convention that $\xi_{0}=1$.

The structure of the motivic Steenrod algebra at odd primes is essentially the same as the structure of the classical Steenrod algebra. In fact, in the odd primary case, the motivic Steenrod algebra is the classical Steenrod algebra tensored over $\mathbb{F}_{p}$ with a larger coefficient ring. Consequently, motivic Ext calculations over $A_{*}$ are essentially identical to classical Ext calculations.

The situation at $p=2$ is more interesting. In this case, the dual motivic Steenrod algebra $A_{*}$ is the commutative $\mathbb{M}_{2}$-algebra generated by elements $\tau_{i}$ for $i \geq 0$ and $\xi_{j}$ for $j \geq 1$, subject to the relations

$$
\tau_{i}^{2}=\tau \xi_{i+1}+\rho \tau_{i+1}+\rho \tau_{0} \xi_{i+1} .
$$

Here $\tau_{i}$ has degree $\left(2^{i+1}-1,2^{i}-1\right)$, and $\xi_{j}$ has degree $\left(2^{j+1}-2,2^{j}-1\right)$. The coproduct $\Delta$ on $A_{*}$ is described by

$$
\Delta\left(\tau_{i}\right)=\tau_{i} \otimes 1+\sum_{k=0}^{i} \xi_{i-k}^{2^{k}} \otimes \tau_{k}
$$

and

$$
\Delta\left(\xi_{j}\right)=\sum_{k=0}^{j} \xi_{j-k}^{2^{k}} \otimes \xi_{k} .
$$

As before, we adopt the usual convention that $\xi_{0}=1$.

Remark 2.8. If we invert $\tau$ and set $\rho$ equal to zero, then we obtain the classical Steenrod algebra tensored over $\mathbb{F}_{2}$ with $\mathbb{M}_{2}\left[\tau^{-1}\right]$. The point is that $\xi_{i+1}$ is no longer needed as a generator because $\xi_{i+1}=\tau^{-1} \tau_{i}^{2}$. Then the elements $\tau_{i}$ are generators, with no relations.

Unlike in the classical case, the motivic Steenrod algebra is more accurately a Hopf algebroid (in the sense of [Rav86, Appendix 1], for example). For sake of tradition, we will not use the term "algebroid" in this context. This means that the motivic Steenrod algebra acts non-trivially on the motivic cohomology of a point in general. In other words, the right unit $\eta_{R}: \mathbb{M}_{p} \rightarrow A_{*}$ is not the same as the left unit $\eta_{L}: \mathbb{M}_{p} \rightarrow A_{*}$. More precisely, $\eta_{L}$ is the obvious inclusion, while $\eta_{R}(\tau)=\tau+\rho \tau_{0}$ 
and $\eta_{R}(\rho)=\rho$. This complication occurs only if $\rho=[-1]$ is non-zero in $K_{*}^{M}(k) / p$, i.e., only if -1 is not a $p$ th power in the ground field $k$.

For definiteness, we restate the action of the Steenrod squares on $\mathbb{M}_{p}$ in more concrete terms. At $p=2$, we have $\operatorname{Sq}^{1}(\tau)=\rho$. There are also non-zero squaring operations on other elements, but they can all be derived from this basic formula.

The motivic Cartan formula takes the form

$$
\mathrm{Sq}^{2 k}(x y)=\sum_{a+b=2 k} \tau^{\epsilon} \mathrm{Sq}^{a}(x) \mathrm{Sq}^{b}(y),
$$

where $\epsilon$ is 0 if $a$ and $b$ are even, while $\epsilon$ is 1 if $a$ and $b$ are odd Voe03a, Proposition 9.7]. (There is also a motivic Cartan formula for odd squares, but it has some additional complications involving $\rho$.) This implies that

$$
\operatorname{Sq}^{2}\left(\tau^{2}\right)=\tau \operatorname{Sq}^{1}(\tau) \operatorname{Sq}^{1}(\tau)=\rho^{2} \tau
$$

At odd primes, we have that $\beta(\tau)=\rho$, where $\beta$ is the Bockstein operation.

2.3. The cohomology of the Steenrod algebra. The next step is to compute the cohomology of the motivic Steenrod algebra, i.e.,

$$
\operatorname{Ext}_{k}=\operatorname{Ext}_{A}\left(\mathbb{M}_{p}, \mathbb{M}_{p}\right) \text {. }
$$

This object is a trigraded commutative ring with higher structure in the form of Massey products. Two of the gradings correspond to the degrees familiar in the classical situation. The additional grading corresponds to the motivic weight, which has no classical analogue.

The cobar resolution is the standard way to compute Ext groups. This works just as well motivically as it does classically. The cobar resolution is useful for (very) low-dimensional explicit calculations and for general properties. In a larger range, one must attack the calculation with more sophisticated tools such as the May spectral sequence May65 DI10.

In the modern era, the best way to determine Ext in a range is by computer. These entirely algebraic invariants are effectively computable in a large range. Computer algorithms typically rely on minimal resolutions rather than the cobar resolution because it grows more slowly. In practice, computer calculations of Ext far outpace our ability to interpret the data with the Adams spectral sequence Bru89. Bru93 Bru97 Nas [WX].

Of course, explicit calculations over a field $k$ depend on specific knowledge of $K_{*}^{M}(k)$.

Remark 2.9. Over $\mathbb{C}$ and at $p=2$, the cohomology of the motivic Steenrod algebra was computed with the motivic May spectral sequence through the 70-stem [Isa]. The most recent computer calculations over $\mathbb{C}$ extend far beyond the 100-stem, with ongoing progress into even higher stems [IWX].

Remark 2.10. Over $\mathbb{F}_{q}$ and at $p=2$, the cohomology of the motivic Steenrod algebra is studied in detail through the 20-stem [WØ17. The Milnor $K$-theory $K_{*}^{M}\left(\mathbb{F}_{q}\right)$ is relatively simple, as described in Example 2.6 this makes explicit computations practical.

If $\operatorname{char} \mathbb{F}_{q} \equiv 1 \bmod 4$, then -1 is a square and $\rho$ is zero in $\mathbb{M}_{2}$. Therefore, the situation is essentially identical to the calculation over $\mathbb{C}$.

However, if $\operatorname{char} \mathbb{F}_{q} \equiv 3 \bmod 4$, then $\rho$ is non-zero in $\mathbb{M}_{2}$. This case is more complicated (and more interesting). 
2.4. The $\rho$-Bockstein spectral sequence. Over $\mathbb{R}$, the cohomology $\mathbb{M}_{2}$ of a point is $\mathbb{F}_{2}[\tau, \rho]$. As discussed in Section 2.2, the motivic Steenrod algebra acts non-trivially on a point. This complication significantly increases the difficulty of Ext calculations. In other terms, the relation $\tau_{i}^{2}=\tau \xi_{i+1}+\rho \tau_{i+1}+\rho \tau_{0} \xi_{i+1}$ is the source of these difficulties.

In this type of situation, the standard approach is to impose a filtration that hides these complications (or rather, pushes them into higher filtration). Then one obtains a spectral sequence that computes Ext.

In this case, we filter by powers of $\rho$ and obtain a $\rho$-Bockstein spectral sequence that converges to $\operatorname{Ext}_{\mathbb{R}}$ Hil11] DI17. The associated graded object of $A_{*}^{\mathbb{R}}$ is easily identified with $\mathbb{A}_{*}^{\mathbb{C}}[\rho]$. From this observation, one can deduce that the spectral sequence takes the form

$$
\operatorname{Ext}_{\mathbb{C}}[\rho] \Rightarrow \operatorname{Ext}_{\mathbb{R}}
$$

In order for the $\rho$-Bockstein spectral sequence to be practical, one needs a method for computing differentials. The first step in this program is to consider the effect of inverting $\rho$ [DI17, Theorem 4.1]. If one inverts $\rho$ in $A_{*}$, then the relation

$$
\tau_{i}^{2}=\tau \xi_{i+1}+\rho \tau_{i+1}+\rho \tau_{0} \xi_{i+1}
$$

can be rewritten in the form

$$
\tau_{i+1}=\rho^{-1} \tau_{i}^{2}+\rho^{-1} \tau \xi_{i+1}+\tau_{0} \xi_{i+1} .
$$

This shows that $A_{*}\left[\rho^{-1}\right]$ can be simply described as $\mathbb{M}_{2}\left[\rho^{-1}\right]\left[\tau_{0}, \xi_{1}, \xi_{2}, \ldots\right]$. The cohomology of $A_{*}\left[\rho^{-1}\right]$ is then straightforward to compute. One obtains the isomorphism

$$
\operatorname{Ext}_{\mathbb{R}}\left[\rho^{-1}\right] \cong \operatorname{Ext}_{\text {cl }}\left[\rho^{ \pm 1}\right] .
$$

In other words, the $\rho$-periodic part of $\operatorname{Ext}_{\mathbb{R}}$ is identified with classical Ext groups. Beware that this isomorphism changes degrees. For example, the motivic element $h_{i+1}$ in the $\left(2^{i+1}-1\right)$-stem maps to the classical element $h_{i}$ in the $\left(2^{i}-1\right)$-stem.

Remark 2.11. In fact, this computational observation extends to an equivalence of homotopy categories. Namely, the $\rho$-periodic $\mathbb{R}$-motivic stable homotopy category $\mathbf{S H}(\mathbb{R})\left[\rho^{-1}\right]$ is equivalent to the classical stable homotopy category [Bac18].

Inverting $\rho$ on an $\mathbb{R}$-motivic spectrum is analogous to taking the geometric fixed points of a $C_{2}$-equivariant spectrum.

It remains to compute the $\rho^{r}$-torsion in $\operatorname{Ext}_{\mathbb{R}}$ for all $r$, i.e., to compute the $\rho$ Bockstein $d_{r}$ differentials for all $r$. It turns out that these differentials are forced by combinatorial considerations in a large range. Every element $x$ of the $\rho$-Bockstein $E_{1}$-page falls into one of three categories:

(1) $x$ survives, and $\rho^{k} x$ is not hit by any differential for all $k$. These elements are completely known because of the calculation of $\operatorname{Ext}_{\mathbb{R}}\left[\rho^{-1}\right]$.

(2) $x$ survives and $\rho^{k} x$ is hit by some differential for some $k \geq 1$.

(3) $x$ does not survive.

Somewhat surprisingly, these considerations determine many $\rho$-Bockstein differentials. In other words, in a large range, there is only one pattern of differentials that is consistent with sorting all elements into these three classes.

See [DI17, Section 5] for a description of this process in low dimensions. Preliminary results indicate that the same naive method works into much higher dimensions. 
Remark 2.12. The $\rho$-Bockstein spectral sequence is useful for fields other than $\mathbb{R}$, in which -1 is not a square and therefore $\rho$ is non-zero. Some examples are finite fields $\mathbb{F}_{q}$ for which char $\mathbb{F}_{q} \equiv 3 \bmod 4$ WØ17.

2.5. The motivic Adams spectral sequence. The point of computing $\operatorname{Ext}_{k}$ is that it serves as the input to the motivic Adams spectral sequence

$$
E_{2}=\operatorname{Ext}_{k} \Rightarrow \pi_{*, *}^{k} \text {. }
$$

We will not give a detailed construction of the spectral sequence. Just as in the classical case, the idea is to construct an Adams resolution of the motivic sphere spectrum, using copies of the motivic Eilenberg-Mac Lane $\mathbf{M} \mathbb{F}_{2}$ and a few of its basic properties [Mor99a] DI10] HKO11a].

A certain amount of care must be taken with convergence of the motivic Adams spectral sequence. In particular, one must complete not only with respect to a prime, but also with respect to the first Hopf map $\eta$. Convergence results are worked out in HKO11a] HKO11b Man18] [KW18, and we will not discuss them further here.

There are three major phases to carrying out an Adams spectral sequence computation:

(1) Compute the $E_{2}$-page. This is an algebraic problem. See Section 2.3.

(2) Compute Adams differentials. This is the hardest of the three steps.

(3) The $E_{\infty}$-page gives the filtration quotients in a filtration on $\pi_{*, *}$, but these quotients can hide some of the structure of $\pi_{*, *}$. We may have two elements $\alpha$ and $\beta$ of $\pi_{*, *}$ detected by elements $a$ and $b$ of the $E_{\infty}$-page with filtrations $i$ and $j$ respectively. Sometimes, the product $\alpha \beta$ is non-zero in $\pi_{*, *}$, but is detected in filtration greater than $i+j$. In this case, the product $a b$ equals zero in the $E_{\infty}$-page, even though $\alpha \beta$ is non-zero. Some work is required to reconstruct $\pi_{*, *}$ itself from its filtration quotients. See [Isa, Section 4.1.1] for a careful discussion of these issues.

\section{The MotiviC EFFECTIVE SLICE SPECTRAL SEQUenCE}

In this section we review the effective slice filtration of the stable motivic homotopy category $\mathbf{S H}(k)$. This filtration was originally proposed by Voevodsky Voe02a under the name "slice filtration". The goal is to understand the associated spectral sequence for the sphere. The latter approximates the stable motivic homotopy groups in a way we will make precise.

The effective slice spectral sequence (3.5) and its very effective version introduced in $\mathrm{S \varnothing 12}$ are algebro-geometric analogues of the topological Atiyah-Hirzebruch spectral sequence AH61. Among the applications of the effectice filtration we note Voevodsky's construction of the effective slice spectral sequence relating motivic cohomology to algebraic $K$-theory [Voe02b], Levine's proof of full faithfulness of the constant functor from the stable topological homotopy category to the stable motivic homotopy category over algebraically closed fields [Lev14, a new proof of Milnor's conjecture on quadratic forms in [RØ16], and the identification of Morel's plus part of the rational sphere spectrum with rational motivic cohomology along with finiteness for the motivic stable homotopy groups over finite fields in RSØa.

The equivariant slice filtration has become an important tool classical homotopy theory. For example, it is central to major progress on elements of Kervaire invariant one [HHR16. The reader should beware that the equivariant slice filtration does not 
directly correspond to the motivic effective slice filtration. However, the filtrations do happen to align in many cases related to $K$-theory and cobordism.

3.1. The effective slice filtration. Let $\mathbf{S} \mathbf{H}^{\text {eff }}(k)$ be the localizing subcategory of the stable motivic homotopy category $\mathbf{S H}(k)$ generated by the motivic suspension spectra of all smooth schemes over $k$, i.e., the smallest full triangulated subcategory of $\mathbf{S H}(k)$ that contains suspension spectra of smooth schemes and is closed under coproducts Voe02a]. These subcategories form the effective slice filtration

$$
\cdots \subset \Sigma^{2 q+2, q+1} \mathbf{S H}^{\text {eff }}(k) \subset \Sigma^{2 q, q} \mathbf{S H}^{\text {eff }}(k) \subset \Sigma^{2 q-2, q-1} \mathbf{S H}^{\text {eff }}(k) \subset \cdots .
$$

Since $\mathbf{S H}^{\text {eff }}(k)$ is closed under simplicial suspsension and desuspension, the subcategory $\Sigma^{2 q, q} \mathbf{S H}^{\mathrm{eff}}(k)$ is equal to the subcategory $\Sigma^{0, q} \mathbf{S H}^{\mathrm{eff}}(k)$. The effective slice filtration is exhaustive in the sense that $\mathbf{S H}(k)$ is the smallest triangulated subcategory that is closed under coproducts and contains $\Sigma^{2 q, q} \mathbf{S H}^{\mathrm{eff}}(k)$ for all $q$.

The inclusion $\Sigma^{2 q, q} \mathbf{S H}^{\text {eff }}(k) \subset \mathbf{S H}(k)$ is left adjoint to a functor that takes a motivic spectrum to its " $q$ th effective slice cover". (This is analogous to the classical inclusion of $q$-connected spectra into all spectra, which is left adjoint to the $q$-connected cover.) We write $\mathrm{f}_{q}$ for the functor that takes a motivic spectrum to its $q$ th effective slice cover.

Any motivic spectrum $\mathbf{E}$ has an effective slice filtration

$$
\cdots \rightarrow \mathrm{f}_{q+1} \mathbf{E} \rightarrow \mathrm{f}_{q} \mathbf{E} \rightarrow \mathrm{f}_{q-1} \mathbf{E} \rightarrow \cdots .
$$

The associated graded object of this filtration is $\mathbf{s}_{*} \mathbf{E}$, where the $q$ th component $\mathbf{s}_{q} \mathbf{E}$ is the cofiber of $\mathrm{f}_{q+1} \mathbf{E} \rightarrow \mathrm{f}_{q} \mathbf{E}$.

A related construction employs the very effective subcategory $\mathbf{S H}^{\text {veff }}(k)$ introduced in [SØ12, §5]. This is the smallest full subcategory of $\mathbf{S H}(k)$ that contains all suspension spectra of smooth schemes of finite type, is closed under homotopy colimits, and is closed under extensions in the sense that if $X \rightarrow Y \rightarrow Z$ is a cofiber sequence such that $X$ and $Z$ belong to $\mathbf{S H}^{\text {veff }}(k)$, then so does $Y$.

We note that $\mathbf{S} \mathbf{H}^{\text {veff }}(k)$ is contained in $\mathbf{S} \mathbf{H}^{\text {eff }}(k)$ but it is not a triangulated subcategory of $\mathbf{S H}(k)$ since it is not closed under simplicial desuspension. The very effective slice filtration takes the form

$$
\cdots \subset \Sigma^{2 q+2, q+1} \mathbf{S H}^{\mathrm{eff}}(k) \subset \Sigma^{2 q, q} \mathbf{S H}^{\mathrm{eff}}(k) \subset \Sigma^{2 q-2, q-1} \mathbf{S H}^{\mathrm{eff}}(k) \subset \cdots,
$$

and we obtain notions of very effective slice covers $\tilde{\mathrm{f}}_{q}$ and very effective slices $\tilde{\mathbf{s}}_{q}$ for all $n \in \mathbb{Z}$. To connect this to motivic homotopy groups we mention the fact that if $\mathbf{E} \in \mathbf{S H}^{\mathrm{veff}}(k)$ then $\pi_{m, n} \mathbf{E}=0$ for $m<n$ [SØ12, Lemma 5.10]. Some examples of very effective motivic spectra are algebraic cobordism MGL, the effective slice cover $\mathbf{k g l}:=\mathrm{f}_{0} \mathbf{K G L}$ of algebraic $K$-theory [SØ12], and the very effective slice cover $\mathbf{k q}:=\tilde{\mathrm{f}}_{0} \mathbf{K Q}$ of hermitian $K$-theory [ARØ17. One advantage of the very effective slice filtration is that it maps to the topological Postnikov tower under Betti realization GRSØ12, §3.3].

Higher structural properties of motivic spectra such as $A_{\infty}$ - and $E_{\infty}$-structures interact well with these filtrations. In GRSØ12 it is shown that if $\mathbf{E}$ is an $A_{\infty}$ - or $E_{\infty}$-algebra in motivic symmetric spectra [Jar00] then $\mathrm{f}_{0} \mathbf{E}$ and $\tilde{\mathrm{f}}_{0} \mathbf{E}$ are naturally equipped with the structure of an $A_{\infty}$ - resp. $E_{\infty}$-algebra. Moreover, the canonical maps $\mathrm{f}_{0} \mathbf{E} \rightarrow \mathbf{E}$ and $\tilde{\mathrm{f}}_{0} \mathbf{E} \rightarrow \mathbf{E}$ can accordingly be modelled as maps of $A_{\infty^{-}}$and $E_{\infty^{-}}$ algebras. For every $q \in \mathbb{Z}$ the functors $\mathrm{f}_{q}, \mathbf{s}_{q}, \tilde{\mathrm{f}}_{q}$, and $\tilde{\mathbf{s}}_{q}$ respect module structures over $E_{\infty}$-algebras. 
Applying motivic homotopy groups to the filtration (3.2) yields by standard techniques an exact couple and the effective slice spectral sequence

$$
E_{m, q, n}^{1}(\mathbf{E})=\pi_{m, n} \mathrm{~s}_{q} \mathbf{E} \Longrightarrow \pi_{m, n} \mathbf{E} .
$$

For calculations it is important to note that the first effective slice differential is induced by the composition $\mathrm{s}_{q} \mathbf{E} \rightarrow \Sigma^{1,0} \mathrm{f}_{q+1} \mathbf{E} \rightarrow \Sigma^{1,0} \mathbf{s}_{q+1} \mathbf{E}$.

3.2. The slices of 1 . By RSØa , the effective slice spectral sequence for the sphere 1 over any field has $E_{1}$-page given by

$$
E_{m, q, n}^{1}=\pi_{m, n} \mathrm{~s}_{q}(\mathbf{1}) .
$$

This spectral sequence converges conditionally, in the sense of Boardman Boa99] to the $\eta$-completed stable motivic homotopy groups. Technically, there is no need to complete at a prime, i.e., the effective slice spectral sequence is defined integrally. However, for practical purposes it is best to further complete one prime at a time. Here $\mathrm{s}_{q}(\mathbf{1})$ is the $q$ th slice of $\mathbf{1}$, i.e., the $q$ th layer of the effective slice filtration on 1.

The first step in understanding the effective slice spectral sequence (3.5) is to understand the slices $\mathbf{s}_{q}(\mathbf{1})$. Voevodsky Voe02a, Conjectures 9 and 10] predicted correctly that these slices are governed by the structure of the Adams-Novikov $E_{2}$ page (see (3.7) below). It turns out that $\mathrm{s}_{0}(\mathbf{1})$ identifies with the integral motivic Eilenberg-Mac Lane spectrum $\mathbf{M} \mathbb{Z}$, while $\mathbf{s}_{q}(\mathbf{1})$ for $q \geq 1$ are $\mathbf{s}_{0}(\mathbf{1})$-modules, i.e., MZZ-modules.

We will now sketch how the slices $\mathrm{s}_{q}(\mathbf{1})$ can be computed. First, a geometric argument shows that the cone of the unit map $\mathbf{1} \rightarrow$ MGL lies in $\Sigma^{2,1} \mathbf{S H}^{\mathrm{eff}}(k)$. It follows that $\mathbf{s}_{0}(\mathbf{1}) \rightarrow \mathrm{s}_{0}$ (MGL) is an isomorphism since $\mathbf{s}_{0}$ is trivial on $\Sigma^{2,1} \mathbf{S H}^{\text {eff }}(k)$.

The slices of MGL are described in Theorem 3.2. It turns out to be more convenient to describe all the slices of MGL simultaneously, rather than one at a time. So we will describe the entire graded motivic spectrum $\mathbf{s}_{*}$ MGL. We use the term "effective slice degree" to refer to this external grading.

Remark 3.1. For the following discussion, if the base field $k$ is of positive characteristic, one must invert its characteristic in the motivic spectra MGL and MZZ. See [R Øa, §2.1-2.2] for precise statements. For legibility we will not make any notational changes.

Theorem 3.2. [Spi10, Corollary 4.7] Hoy15, §8.3] [Spi14, Theorem 3.1] Over a base field of characteristic zero the graded motivic spectrum $\mathbf{s}_{*} \mathbf{M G L}$ is equal to $\mathbf{M} \mathbb{Z}\left[x_{1}, x_{2}, \ldots\right]$, where $x_{j}$ has motivic degree $(2 j, j)$ and effective slice degree $j$.

The expression $\mathbf{M} \mathbb{Z}\left[x_{1}, x_{2}, \ldots\right]$ in Theorem 3.2 bears some explanation. This object is a direct sum of copies of suspensions $\mathbf{M} \mathbb{Z}$, indexed by monomials in the $x_{j}$. Each monomial $x_{1}^{i_{1}} x_{2}^{i_{2}} \ldots x_{r}^{i_{r}}$ contributes a copy of $\Sigma^{2 q, q} \mathbf{M} \mathbb{Z}$ to $\mathbf{s}_{q} \mathbf{M G L}$, where $q=\sum_{j=1}^{r} j i_{j}$. Note that $q$ is the degree of the monomial of the same name in $\pi_{*} \mathbf{M U}=\mathbb{Z}\left[x_{1}, x_{2}, \ldots\right]$, where $\mathbf{M U}$ is the classical complex cobordism spectrum and $x_{j}$ has degree $2 j$ Mil60.

The positive slices of $\mathbf{1}$ are determined in several steps, starting with the standard cosimplicial MGL-resolution

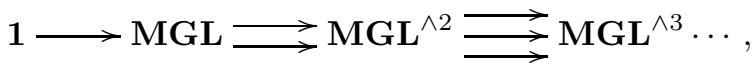


which induces a natural isomorphism

$$
\mathrm{s}_{q}(\mathbf{1}) \stackrel{\sim}{\rightrightarrows} \operatorname{holim}_{\Delta} \mathrm{s}_{q}\left(\mathbf{M G L}^{\wedge(\bullet+1)}\right) .
$$

In order to describe the slices of $\mathbf{M G L}{ }^{\wedge n}$, first recall the smash product decompositions

$$
\mathbf{M G L} \wedge \mathbf{M G L}=\mathbf{M G L}\left[b_{1}, b_{2}, b_{3}, \ldots\right]
$$

and

$$
\mathbf{M U} \wedge \mathbf{M U}=\mathbf{M U}\left[b_{1}, b_{2}, b_{3}, \ldots\right]
$$

from [NSØ09, Lemma 6.4(i)] and Ada74, p. 87] respectively. Here $b_{j}$ is the standard choice of generator in the dual Landweber-Novikov algebra Rav86, Theorem 4.1.11], and the motivic degree of $b_{j}$ is $(2 j, j)$, while the classical degree of $b_{j}$ is $2 j$. These decompositions extend to higher smash powers in an obvious way.

From Theorem 3.2 and these smash product decompositions, it follows that the graded motivic spectrum $\mathbf{s}_{*}\left(\mathbf{M G L}{ }^{\wedge n}\right)$ is $\mathbf{M} \mathbb{Z} \otimes \pi_{*}\left(\mathbf{M} \mathbf{U}^{\wedge n}\right)$ Voe02a, Conjecture $6]$. More precisely, $\pi_{*}\left(\mathbf{M} \mathbf{U}^{\wedge n}\right)$ is a free abelian group on a set of generators, concentrated in even degrees. Each generator of $\pi_{2 q}\left(\mathbf{M} \mathbf{U}^{\wedge n}\right)$ contributes a summand $\Sigma^{2 q, q} \mathbf{M} \mathbb{Z}$ to $\mathbf{s}_{q}\left(\mathbf{M G L}^{\wedge n}\right)$.

With additional work detailed in $\mathrm{RS} \varnothing \mathrm{a}, \S 2.2]$, the above produces an isomorphism

$$
\mathrm{s}_{*}(\mathbf{1}) \simeq \mathbf{M} \mathbb{Z} \otimes \operatorname{Ext}_{\mathbf{M U}_{*}, *} \mathbf{M U}\left(\mathrm{MU}_{*}, \mathrm{MU}_{*}\right) .
$$

More precisely, each copy of $\mathbb{Z} / n$ in $\operatorname{Ext}_{\mathbf{M} \mathbf{U}_{*} \mathbf{M U}}^{p, 2 q}\left(\mathbf{M U}_{*}, \mathbf{M U}_{*}\right)$ contributes a summand $\Sigma^{2 q-p, q} \mathbf{M} \mathbb{Z} / n$ to $\mathbf{s}_{q}(\mathbf{1})$. Here $p$ is the Adams-Novikov filtration, i.e., the homological degree of the Ext group, and $2 q$ is the total degree, i.e., the stem plus the Adams-Novikov filtration.

We note the slices are $\mathbf{M} \mathbb{Z}$-modules and the effective slice $d_{1}$-differentials are maps between motivic Eilenberg-MacLane spectra. These facts make the effective slice spectral sequence amenable to calculations over a base scheme affording an explicit description of its motivic cohomology ring along with the action of the motivic Steenrod algebra.

The Ext groups in (3.7) form the $E_{2}$-page of the Adams-Novikov spectral sequence, which has been extensively studied by topologists [Rav86. From an algebraic viewpoint, this is the cohomology of the "Hopf algebroid" corepresenting the functor carrying a commutative ring to the groupoid of formal group laws over it and their strict isomorphisms Ada74]. If $(s, t) \neq(0,0)$, then $\operatorname{Ext}_{\mathbf{M} \mathbf{U}_{*} \mathbf{M U}}\left(\mathbf{M} \mathbf{U}_{*}, \mathbf{M U}_{*}\right)$ is finite.

In practice, $\operatorname{Ext}_{\mathbf{M U}} \mathbf{M U}_{*}\left(\mathbf{M U}_{*}, \mathbf{M U}_{*}\right)$ is most easily studied one prime at a time. For the Brown-Peterson spectrum BP at a prime $p$, we have

$$
\operatorname{Ext}_{\mathbf{M U}}^{s, t} \mathbf{M U}\left(\mathbf{M U}_{*}, \mathbf{M U}_{*}\right)=\bigoplus_{p} \operatorname{Ext}_{\mathbf{B P}_{*} \mathbf{B P}}^{s, t}\left(\mathbf{B P}_{*}, \mathbf{B P}_{*}\right)
$$

when $(s, t) \neq(0,0)$, so one should really study $\operatorname{Ext}_{\mathbf{B} \mathbf{P}_{*} \mathbf{B P}}^{s, t}\left(\mathbf{B P}_{*}, \mathbf{B P}_{*}\right)$ separately for each prime.

This input provides a systematic way of keeping track of the direct summands of $\mathrm{s}_{q}(\mathbf{1})$. For example, at $p=2$, the well-known elements $\alpha_{1}^{q} \operatorname{in}_{\operatorname{Ext}_{\mathbf{B}} \mathbf{P}_{*} \mathbf{B P}^{2,2 q}}\left(\mathbf{B P}_{*}, \mathbf{B P}_{*}\right)$ contribute $\Sigma^{q, q} \mathbf{M} \mathbb{Z} / 2$ summands to $\mathbf{s}_{q}(\mathbf{1})$, and the element $\alpha_{2 / 2}$ in

$$
\operatorname{Ext}_{\mathbf{B P} * \mathbf{B P}}^{1,4}\left(\mathbf{B P}_{*}, \mathbf{B} \mathbf{P}_{*}\right)
$$


contributes a $\Sigma^{3,2} \mathbf{M} \mathbb{Z} / 4$ summand to $\mathrm{s}_{2}(\mathbf{1})$. At $p=3$, the element $\alpha_{1}$ in

$$
\operatorname{Ext}_{\mathbf{B P} \mathbf{P}_{*} \mathbf{B P}}^{1,4}\left(\mathbf{B P}_{*}, \mathbf{B P}_{*}\right)
$$

contributes $\Sigma^{3,2} \mathbf{M} \mathbb{Z} / 3$ to $\mathbf{S}_{2}(\mathbf{1})$. These copies of $\Sigma^{3,2} \mathbf{M} \mathbb{Z} / 4$ and $\Sigma^{3,2} \mathbf{M} \mathbb{Z} / 3$ assemble into a copy of $\Sigma^{3,2} \mathbf{M} \mathbb{Z} / 12$ in $\mathbf{s}_{2}(\mathbf{1})$.

Remark 3.3. Our notation for $\mathbf{s}_{*}(\mathbf{1})$ suggests that we are describing $\mathbf{s}_{*}(\mathbf{1})$ as a ring object, but the notation is somewhat deceptive. For example, consider the map $\mathrm{s}_{1}(\mathbf{1}) \wedge \mathrm{s}_{1}(\mathbf{1}) \rightarrow \mathrm{s}_{2}(\mathbf{1})$ at $p=2$, where $\mathrm{s}_{1}(\mathbf{1})$ is equivalent to $\Sigma^{1,1} \mathbf{M} \mathbb{Z} / 2$ corresponding to $\alpha_{1}$, and $\mathbf{s}_{2}(\mathbf{1})$ is equivalent to

$$
\Sigma^{2,2} \mathbf{M} \mathbb{Z} / 2 \vee \Sigma^{3,2} \mathbf{M} \mathbb{Z} / 4
$$

corresponding to $\alpha_{1}^{2}$ and $\alpha_{2 / 2}$ respectively.

The source $\Sigma^{1,1} \mathbf{M} \mathbb{Z} / 2 \wedge \Sigma^{1,1} \mathbf{M} \mathbb{Z} / 2$ splits as a wedge of suspensions of copies of $\mathbf{M} \mathbb{Z} / 2$, where the wedge is indexed by a basis for the motivic Steenrod algebra. Thus we want to calculate the map

$$
\Sigma^{2,2} \bigvee_{b \in B} \Sigma^{d_{b}} \mathbf{M} \mathbb{Z} / 2 \rightarrow \Sigma^{2,2} \mathbf{M} \mathbb{Z} / 2 \vee \Sigma^{3,2} \mathbf{M} \mathbb{Z} / 4,
$$

where $B$ is a basis for the motivic Steenrod algebra, and $d_{b}$ is the bidegree of $b$.

If we restrict to the summand of the source of (3.8) corresponding to the element 1 of the motivic Steenrod algebra, then we have a map

$$
\Sigma^{2,2} \mathbf{M} \mathbb{Z} / 2 \rightarrow \Sigma^{2,2} \mathbf{M} \mathbb{Z} / 2 \vee \Sigma^{3,2} \mathbf{M} \mathbb{Z} / 4 .
$$

This map is the identity on the first summand of the target and zero on the second summand of the target, corresponding to the multiplicative relation $\alpha_{1} \cdot \alpha_{1}=\alpha_{1}^{2}$.

However, if we restrict to the summand of the source of (3.8) corresponding to the element $\mathrm{Sq}^{1}$ of the motivic Steenrod algebra, then we have a map

$$
\Sigma^{3,2} \mathbf{M} \mathbb{Z} / 2 \rightarrow \Sigma^{2,2} \mathbf{M} \mathbb{Z} / 2 \vee \Sigma^{3,2} \mathbf{M} \mathbb{Z} / 4 .
$$

This map turns out to be non-trivial when restricted to the second summand of the target, and this has explicit computational consequences for the effective slice spectral sequence.

This warning about multiplicative structures applies to other slice calculations in this manuscript.

It is natural to ask how $\eta$ acts on the slices of $\mathbf{1}$. A complete description can be extracted from the work of Andrews-Miller [AM17] that calculates the $\alpha_{1}$-periodic Ext groups over $\mathbf{B} \mathbf{P}_{*} \mathbf{B P}$ at the prime 2 . This shows that $\mathbf{s}_{*}(\mathbf{1})\left[\eta^{-1}\right]$ is

$$
\mathbf{M} \mathbb{Z} / 2\left[\alpha_{1}^{ \pm 1}, \alpha_{3}, \alpha_{4}\right] / \alpha_{3}^{2},
$$

where $\alpha_{1}$ has motivic degree $(1,1)$ and effective slice degree $1 ; \alpha_{3}$ has motivic degree $(5,3)$ and effective slice degree 3 ; and $\alpha_{4}$ has motivic degree $(7,4)$ and effective slice degree 4.

3.3. The slices of other motivic spectra. Related to (3.7), the slices of algebraic $K$-theory KGL, hermitian $K$-theory $\mathbf{K Q}$, and higher Witt-theory $\mathbf{K W}$ can be identified.

Theorem 3.4. Lev08 RØ16

(1) The graded motivic spectrum $\mathbf{s}_{*} \mathbf{K G L}$ is equivalent to $\mathbf{M} \mathbb{Z}\left[\beta^{ \pm 1}\right]$, where $\beta$ has motivic degree $(2,1)$ and effective slice degree 1. 
(2) When $\operatorname{char}(k) \neq 2$, the graded motivic spectrum $\mathbf{s}_{*} \mathbf{K Q}$ is equivalent to

$$
\mathbf{M} \mathbb{Z}\left[\alpha_{1}, v_{1}^{ \pm 2}\right] / 2 \alpha_{1},
$$

where $\alpha_{1}$ has motivic degree $(1,1)$ and effective slice degree 1 , and $v_{1}^{2}$ has motivic degree $(4,2)$ and effective slice degree 2 .

(3) When $\operatorname{char}(k) \neq 2$, the graded motivic spectrum $\mathbf{s}_{*} \mathbf{K W}$ is equivalent to

$$
\mathbf{M} \mathbb{Z} / 2\left[\alpha_{1}^{ \pm 1}, v_{1}^{ \pm 2}\right]
$$

where $\alpha_{1}$ has motivic degree $(1,1)$ and effective slice degree 1 , and $v_{1}^{2}$ has motivic degree $(4,2)$ and effective slice degree 2 .

Part (11) of Theorem 3.4 says that $\mathrm{s}_{q} \mathbf{K G L}$ is $\Sigma^{2 q, q} \mathbf{M} \mathbb{Z}$.

In Part (2) of Theorem 3.4, the monomials $v_{1}^{2 q}$ contribute copies of $\Sigma^{4 q, 2 q} \mathbf{M} \mathbb{Z}$ to $\mathrm{s}_{2 q} \mathbf{K Q}$. Also, for $p \geq 1$, monomials $\alpha_{1}^{p} v_{1}^{2 q}$ contribute copies of $\Sigma^{4 q+p, 2 q+p} \mathbf{M} \mathbb{Z} / 2$ to $\mathbf{s}_{2 q+p} \mathbf{K Q}$. Beware that the notation for $\mathbf{s}_{*} \mathbf{K Q}$ only partly describes the multiplicative structure, as in Remark 3.3 .

We obtain Part (3) of Theorem 3.4 from Part (2) since $\mathbf{K W}$ is obtained from $\mathbf{K Q}$ by inverting $\eta$ in the same way that $\mathbf{1}\left[\eta^{-1}\right]$ is obtained from $\mathbf{1}$.

Remark 3.5. Spitzweck's work on motivic cohomology in Spi12 shows the isomorphisms in (3.7), (3.9), and all three parts of Theorem 3.4 hold not only over fields, but also over Dedekind domains of mixed characteristic with no residue fields of characteristic 2 (see RSØa, §2.3]).

Thanks to the slice calculations reviewed in this section we know precisely how the $E^{1}$-pages of the effective slice spectral sequences for $\mathbf{1}, \mathbf{1}\left[\eta^{-1}\right], \mathbf{K G L}, \mathbf{K Q}$, and KW are given in terms of motivic cohomology groups of the base scheme. Now all the fun can begin with determining the corresponding differentials and resolving the information hidden by the associated graded structure of the effective slice $E_{\infty}$-pages.

\section{4. $\mathbb{C}$-motivic Stable homotopy GRoups}

In this section, we fix the base field $k=\mathbb{C}$. This special case is made easier by the fact that $\mathbb{C}$ has trivial arithmetic properties, i.e., $K_{*}^{M}(\mathbb{C}) / p$ is trivial. In fact, the calculations for any algebraically closed field of characteristic zero work out identically.

To begin, the stable homotopy groups $\pi_{*, 0}^{\mathbb{C}}$ are isomorphic to the classical stable homotopy groups $\pi_{*}$ Lev14. This isomorphism occurs even without completion. However, complicated exotica phenomena occur in other weights.

In this section, we also fix the prime $p=2$. There are interesting phenomena to study at odd primes, but those cases have not yet been studied as extensively. See Sta16] for some $\mathbb{C}$-motivic results at odd primes.

As mentioned in Remark 2.9, the cohomology of the $\mathbb{C}$-motivic Steenrod algebra is completely known in a large range. Current computer calculations extend beyond the 100-stem, with partial information out to the 140-stem. We recommend that the reader view $\mathbb{C}$-motivic Adams charts throughout this discussion [Isa14.

4.1. $\mathbb{C}$-motivic and classical stable homotopy groups. As observed in Remark 2.8, inverting $\tau$ takes the $\mathbb{C}$-motivic Steenrod algebra to the classical Steenrod 
algebra with $\tau^{ \pm 1}$ adjoined. In fact, this principle extends to Ext groups, so that there is an isomorphism

$$
\operatorname{Ext}_{\mathbb{C}}\left(\mathbb{M}_{2}, \mathbb{M}_{2}\right)\left[\tau^{-1}\right] \cong \operatorname{Ext}_{\mathrm{cl}}\left(\mathbb{F}_{2}, \mathbb{F}_{2}\right)\left[\tau^{ \pm 1}\right]
$$

where $\operatorname{Ext}_{\mathrm{cl}}\left(\mathbb{F}_{2}, \mathbb{F}_{2}\right)$ represents Ext groups over the classical Steenrod algebra. Even further, there is an isomorphism between the $\tau$-periodic $\mathbb{C}$-motivic Adams spectral sequence and the classical Adams spectral sequence. Consequently, the $\tau$-periodic $\mathbb{C}$-motivic stable homotopy groups are isomorphic to the classical stable homotopy groups with $\tau^{ \pm 1}$ adjoined.

This comparison between the $\mathbb{C}$-motivic and classical situations is induced by the Betti realization functor that takes a complex variety to its underlying topological space of $\mathbb{C}$-valued points.

4.2. $\eta$-periodic-phenomena. Classically, the element $h_{1}^{4}$ of $\operatorname{Ext}_{\mathrm{cl}}$ is zero. From Section 4.1 it follows that $\mathbb{C}$-motivically, $\tau^{k} h_{1}^{4}$ equals zero for some $k$. Classically, there is a relation $h_{0}^{2} h_{2}=h_{1}^{3}$. In the $\mathbb{C}$-motivic setting, the weight of $h_{0}^{2} h_{2}$ is 2 , while the weight of $h_{1}^{3}$ is 3 . Consequently, the correct motivic relation is $h_{0}^{2} h_{2}=\tau h_{1}^{3}$. Therefore,

$$
\tau h_{1}^{4}=h_{0}^{2} h_{1} h_{2}=0
$$

but it turns out that $h_{1}^{4}$ is not zero. Moreover, every element $h_{1}^{k}$ is non-zero for all $k \geq 0$.

There is a naive explanation for this phenomenon. Recall that the element $h_{1}$ is detected by $\left[\xi_{1}\right]$ in the $\mathbb{C}$-motivic cobar resolution. Unlike in the classical case, the element $\xi_{1}$ is indecomposable in the $\mathbb{C}$-motivic dual Steenrod algebra. It is this property of the $\mathbb{C}$-motivic dual Steenrod algebra that ultimately allows $h_{1}$ to be not nilpotent.

This exotic behavior of $h_{1}$ leads us inevitably to ask about the effect of inverting $h_{1}$ on the cohomology of the $\mathbb{C}$-motivic Steenrod algebra, and about the effect of inverting the first Hopf map $\eta$ in the $\mathbb{C}$-motivic stable homotopy groups. Inspection of a motivic Adams chart Isa14 reveals many classes, such as $c_{0}, d_{0}$, and $e_{0}$ that are non-zero after inverting $h_{1}$.

We draw particular attention to the element $B_{1}$ (also known as $M h_{1}$ ) in the 46stem. From the perspective of the May spectral sequence, it is a surprise that this element survives $h_{1}$-inversion because it is unrelated to any of the many elements in lower stems that survive $h_{1}$-inversion. This observation eventually led to a clean calculation.

Theorem 4.1. GI15 The $h_{1}$-periodic cohomology of the $\mathbb{C}$-motivic Steenrod algebra is

$$
\operatorname{Ext}_{\mathbb{C}}\left[h_{1}^{-1}\right] \cong \mathbb{F}_{2}\left[h_{1}^{ \pm 1}\right]\left[v_{1}^{4}, v_{2}, v_{3}, \cdots\right]
$$

where $v_{1}^{4}$ has degree $(8,4,4)$ and $v_{n}$ has degree $\left(2^{n}-2,1,2^{n-1}-1\right)$.

The article GI15] conjectured that there are Adams differentials $d_{2}\left(v_{n}\right)=h_{1} v_{n-1}^{2}$ for all $n \geq 3$. This pattern of Adams differentials would determine the $\eta$-periodic $\mathbb{C}$ motivic stable homotopy groups. The conjecture was proved by Andrews and Miller AM17, who computed the $\alpha_{1}$-periodic $E_{2}$-page of the Adams-Novikov spectral sequence. This Adams-Novikov computation immediately determines the $\eta$-periodic $\mathbb{C}$-motivic stable homotopy groups, using the circle of ideas discussed below in Section 4.3 . 
Theorem 4.2. AM17] The $\eta$-periodic $\mathbb{C}$-motivic stable homotopy groups are

$$
\mathbb{F}_{2}\left[\eta^{ \pm 1}\right][\mu, \sigma] / \sigma^{2},
$$

where $\mu$ has degree $(9,5)$ and $\sigma$ has degree $(7,4)$.

Theorem 4.2 holds even without completions.

4.3. Adams differentials. The hardest part of a classical or motivic Adams spectral sequence computation is the determination of Adams differentials. Some techniques include:

(1) Relations obtained by shuffling Toda brackets, which then imply that differentials must occur.

(2) Moss's theorem [Mos70, which can show that certain elements in the Adams spectral sequence must survive to detect certain Toda brackets.

(3) Bruner's theorem on the interaction between algebraic squaring operations and Adams differentials Bru84.

(4) Comparison to other information about stable homotopy groups, such as the image of $J$ [Ada66], the homotopy groups of tmf [DFHH14], and the Adams-Novikov spectral sequence.

Many examples of these techniques appear in BMT70 Bru84 Isa IX15] [MT67 [WX]. The manuscript Isa contains an exhaustive discussion of Adams differentials up to the 59-stem. With much effort, this bound was pushed slightly further to the 61-stem [WX]. However, all of these techniques become impractical in higher stems.

Recent work on the comparison between the $\mathbb{C}$-motivic and classical stable homotopy categories has provided a new tool for computing Adams differentials that has allowed us to extend computations into a much larger range IWX. Current calculations extend beyond the 90-stem, with ongoing progress into even higher stems.

Two new ingredients allow for this program to succeed. The first is computer data for the Adams-Novikov $E_{2}$-page. The second ingredient is the following theorem.

Theorem 4.3. Ghe GWX18] Pst18] In $\mathbb{C}$-motivic stable homotopy theory,

(1) The cofiber $\mathbf{1} / \tau$ of $\tau$ is an $E_{\infty}$-ring spectrum, in an essentially unique way.

(2) With suitable finiteness conditions, the homotopy category of $\mathbf{1} / \tau$-modules is equivalent to the derived category of $\mathbf{B} \mathbf{P}_{*} \mathbf{B P}$-comodules.

(3) The Adams spectral sequence for $\mathbf{1} / \tau$ is isomorphic to the algebraic Novikov spectral sequence [Nov67] Mil75] that converges to the Adams-Novikov $E_{2}$ page.

It is remarkable and unexpected that a homotopical category such as $1 / \tau$ modules would have a purely algebraic description. The proof of part (3) of Theorem 4.3 is that the filtrations associated to the two spectral sequences correspond under the equivalence of part (2).

A new, more powerful approach to computing Adams differentials is summarized in the following steps:

(1) Compute the cohomology of the $\mathbb{C}$-motivic Steenrod algebra by machine.

(2) Compute by machine the algebraic Novikov spectral sequence, including all differentials and multiplicative structure. 
(3) Use Theorem 4.3 to completely describe the motivic Adams spectral sequence for $1 / \tau$.

(4) Use the cofiber sequence

$$
\Sigma^{0,-1} \mathbf{1} \stackrel{\tau}{\rightarrow} \mathbf{1} \rightarrow \mathbf{1} / \tau \rightarrow \Sigma^{1,-1} \mathbf{1}
$$

and naturality of Adams spectral sequences to pull back and push forward Adams differentials for $\mathbf{1} / \tau$ to Adams differentials for the motivic sphere.

(5) Apply a variety of ad hoc arguments to deduce additional Adams differentials for the motivic sphere, as described at the beginning of this section.

(6) Use a long exact sequence in homotopy groups to deduce hidden $\tau$ extensions in the motivic Adams spectral sequence for the sphere.

(7) Invert $\tau$ to obtain the classical Adams spectral sequence and the classical stable homotopy groups.

The weak link in this algorithm is step (5), where ad hoc arguments come into play. The method will continue to calculate new stable homotopy groups until the ad hoc arguments become too complicated to resolve. It is not yet clear when this will occur.

4.4. Motivic nilpotence and periodicity. Section 4.2 discussed the non-nilpotence of the element $\eta$ of $\pi_{1,1}^{\mathbb{C}}$. This non-nilpotent behavior led Haynes Miller to propose that there might be an infinite family of periodicity operators $w_{n}$ such that $w_{0}$ is $\eta$, in analogy to the $v_{n}$-periodicity operators that begin with the non-nilpotent element $v_{0}=2$.

In fact, Miller's guess turned out to be correct And18 Ghe17. In more detail, there exist $\mathbb{C}$-motivic ring spectra $\mathbf{K}\left(w_{n}\right)$ whose motivic stable homotopy groups are of the form $\mathbb{F}_{2}\left[w_{n}^{ \pm 1}\right]$. Also, for each $n$, there exist finite $\mathbb{C}$-motivic complexes that possess $w_{n}$-self maps. More precisely, there exist complexes $\mathbf{X}_{n}$ equipped with maps of the form

$$
\Sigma^{d\left|w_{n}\right|} \mathbf{X}_{n} \stackrel{f_{n}}{\longrightarrow} \mathbf{X}_{n}
$$

that induce isomorphisms in $\mathbf{K}\left(w_{n}\right)$-homology. One can then study $w_{n}$-periodic families of elements in $\pi_{*, *}^{\mathbb{C}}$ by considering compositions of the form

$$
\Sigma^{p, q} \mathbf{1} \longrightarrow \Sigma^{d m\left|w_{n}\right|} \mathbf{X}_{n} \stackrel{f_{n}}{\longrightarrow} \cdots \stackrel{f_{n}}{\longrightarrow} \mathbf{X}_{n} \longrightarrow \mathbf{1}
$$

Andrews And18 found the first explicit examples of $w_{1}$-periodic families.

It turns out that there are even more exotic $\mathbb{C}$-motivic periodicities Kra18. Roughly speaking, these periodicities correspond to the elements $h_{i j}$ of the May spectral sequence for $i \geq j$. The $v_{n}$-periodicities correspond to the elements $h_{n+1,0}$, while the $w_{n}$-periodicities correspond to $h_{n+1,1}$. While there do exist $\mathbb{C}$-motivic spectra of the form $\mathbf{K}\left(h_{i j}\right)$, these objects do not all possess ring structures.

\section{5. $\mathbb{R}$-MOTIVIC STABLE HOMOTOPY GROUPS}

In this section, we fix the base field $k=\mathbb{R}$. This special case introduces new phenomena not seen in the $\mathbb{C}$-motivic situation. The first complication is that $K_{*}^{M}(\mathbb{R}) / 2$ is now a polynomial algebra on one class $\rho$. Studying the $\mathbb{R}$-motivic case allows us to grapple with the difficulties presented by the non-zero element $\rho$. However, it avoids even further complications that would be created by relations involving $\rho$. 
In this section, we also fix the prime $p=2$. The $\mathbb{R}$-motivic case at odd primes is not so interesting, since $K_{*}^{M}(\mathbb{R}) / p$ is zero.

As mentioned in Section 2.4. the $\rho$-Bockstein spectral sequence is an effective tool for computing the cohomology of the $\mathbb{R}$-motivic Steenrod algebra. Preliminary work shows that the spectral sequence can be completely analyzed in a large range. See [DI17] for a sense of the structure of the calculation, although the range studied there is just a tiny portion of what is possible.

5.1. $\eta$-periodic phenomena. Just as in the $\mathbb{C}$-motivic case, one can study the effect of inverting $h_{1}$ on the cohomology of the $\mathbb{R}$-motivic Steenrod algebra, and about the effect of inverting the first Hopf map $\eta$ in the $\mathbb{R}$-motivic stable homotopy groups. This study was carried out to completion in GI16.

The first step is to consider the $h_{1}$-periodic version of the $\rho$-Bockstein spectral sequence discussed in Section 2.4, which takes the form

$$
\operatorname{Ext}_{\mathbb{C}}[\rho]\left[h_{1}^{-1}\right] \Rightarrow \operatorname{Ext}_{\mathbb{R}}\left[h_{1}^{-1}\right] .
$$

This spectral sequence can be completely analyzed.

The next step is to consider the $h_{1}$-periodic version of the $\mathbb{R}$-motivic Adams spectral sequence, which takes the form

$$
\operatorname{Ext}_{\mathbb{R}}\left[h_{1}^{-1}\right] \Rightarrow \pi_{*, *}^{\mathbb{R}}\left[\eta^{-1}\right] .
$$

Once again, this spectral sequence can be completely analyzed, but it is much more interesting than the $\mathbb{C}$-motivic case discussed in Section 4.2, which collapses at the $E_{3}$-page. In fact, in this case there are non-trivial differentials on every page of the spectral sequence. These differentials are deduced by analyzing higher homotopical structure, i.e., Toda brackets.

Before we can state the conclusion of the calculation, we need some additional notation. This complication arises because we want to invert an element $\eta$ that has non-zero degree $(1,1)$. We write $\Pi_{m}^{\mathbb{R}}$ for the direct sum

$$
\bigoplus_{n \in \mathbb{Z}} \pi_{m+n, n}^{\mathbb{R}}
$$

which we call the $m$ th Milnor-Witt stem. Then $\eta$ acts on each $\Pi_{m}^{\mathbb{R}}$, and we can consider $\Pi_{m}^{\mathbb{R}}\left[\eta^{-1}\right]$.

Theorem 5.1. GI16] The $\eta$-periodic $\mathbb{R}$-motivic stable homotopy groups $\pi_{*, *}\left[\eta^{-1}\right]$ are given by:

(1) $\Pi_{0}^{\mathbb{R}}\left[\eta^{-1}\right]=\mathbb{Z}_{2}\left[\eta^{ \pm 1}\right]$.

(2) $\Pi_{4 m-1}^{\mathbb{R}}\left[\eta^{-1}\right]=\mathbb{Z} / 2^{u+1}\left[\eta^{ \pm 1}\right]$ for $m>1$, where $u$ is the 2 -adic valuation of $4 m$.

(3) $\Pi_{m}^{\mathbb{R}}\left[\eta^{-1}\right]=0$ otherwise.

The groups that appear in Theorem 5.1 are reminiscent of the groups that appear in the classical image of $J$. One might speculate on a more direct connection between these $\eta$-periodic $\mathbb{R}$-motivic stable homotopy groups and the image of $J$. However, the Toda bracket structures are different.

\section{Motivic Stable homotopy Groups over General FiELdS}

Sections 4 and 5 discussed what is known about $\mathbb{C}$-motivic and $\mathbb{R}$-motivic stable homotopy groups. We now consider motivic stable homotopy groups over larger 
classes of fields. Naturally, specific information is harder to obtain when the base field is allowed to vary widely.

In addition to the phenomena described in the following sections, we also mention the article DI13, which discusses the existence of motivic Hopf maps and some relations satisfied by these maps.

6.1. Motivic graded commutativity. In the classical case, the stable homotopy groups $\pi_{*}$ are graded commutative, in the sense that

$$
\alpha \beta=(-1)^{|\alpha||\beta|} \beta \alpha
$$

for all $\alpha$ and $\beta$. In other words, $\alpha$ and $\beta$ anti-commute if they are both odddimensional classes, and they strictly commute otherwise. Ultimately, the graded commutativity arises from the fact that the twist map

$$
S^{1} \wedge S^{1} \rightarrow S^{1} \wedge S^{1}
$$

has degree -1 .

Remark 6.1. The graded commutativity of the classical stable homotopy groups has non-trivial consequences for the structure of the Adams spectral sequence. For example, consider the third Hopf map $\sigma$ in $\pi_{7}$, which is detected by $h_{3}$ in the Adams spectral sequence. Graded commutativity implies that $2 \sigma^{2}$ must equal zero in $\pi_{14}$. Now $h_{0} h_{3}^{2}$ detects $2 \sigma^{2}$, and it is non-zero in the Adams $E_{2}$-page. Therefore, there must be an Adams differential $d_{2}\left(h_{4}\right)=h_{0} h_{3}^{2}$. This is the first differential in the Adams spectral sequence.

In the motivic situation, graded commutativity takes a slightly more complicated form. The twist map

$$
S^{1,0} \wedge S^{1,0} \rightarrow S^{1,0} \wedge S^{1,0}
$$

represents -1 in $\pi_{0,0}^{k}$, but the twist map

$$
S^{1,1} \wedge S^{1,1} \rightarrow S^{1,1} \wedge S^{1,1}
$$

represents a different element, usually called $\epsilon$, in $\pi_{0,0}^{k}$. This description of the twist maps leads to the following theorem on motivic graded commutativity.

Theorem 6.2. [Dug14, Proposition 1.18] [DI13, Proposition 2.5] [Mor04a, Corollary 6.1.2] Let $k$ be any base field. For $\alpha$ in $\pi_{a, b}^{k}$ and $\beta$ in $\pi_{c, d}^{k}$,

$$
\alpha \beta=(-1)^{(a-b)(c-d)} \epsilon^{b d} \beta \alpha .
$$

Remark 6.3. Classically, the element 2 of $\pi_{0}$ plays the role of the "zeroth Hopf map". Motivically, it is $1-\epsilon$ that plays this role. The cofiber of $1-\epsilon$ is a 2 -cell complex. In the cohomology of this 2-cell complex, there is a $\mathrm{Sq}^{1}$ operation that connects the two cells. In the cofiber of 2 , there is a $\mathrm{Sq}^{1}$ operation, but there is also a non-trivial $\mathrm{Sq}^{2}$ operation when $\rho$ is non-zero.

6.2. Milnor-Witt $K$-theory. In this section, we will recall the work of Morel on the motivic stable homotopy groups $\pi_{n, n}^{k}$ over arbitrary base fields $k$. For more details, see [Mor04a Mor04b] Mor12]. For related work, see also [DK18] Hor18, Appendix] Nes18, Tho16.

Definition 6.4. The Milnor-Witt $K$-theory $K_{*}^{M W}(k)$ of a field $k$ is the graded associative ring generated by elements $[u]$ for all $u$ in $k^{\times}$, and the element $\eta$, subject to the relations 
(1) $[u][1-u]=0$ for all $u$ in $k$ except for 0 and 1 .

(2) $[u v]=[u]+[v]+\eta[u][v]$ for all $u$ and $v$ in $k^{\times}$.

(3) $[u] \eta=\eta[u]$ for all $u$ in $k^{\times}$.

(4) $\eta(\eta[-1]+2)=0$.

The degree of $[u]$ is 1 , and the degree of $\eta$ is -1 .

Setting $\eta=0$ in Milnor-Witt $K$-theory recovers ordinary Milnor $K$-theory (see Section [2.1), so we can view Milnor-Witt $K$-theory as a kind of deformation of Milnor $K$-theory. The first relation in Definition 6.4 is precisely the Steinberg relation, while the second relation is analogous to the additivity relation in Milnor $K$-theory, with an error term involving $\eta$.

Theorem 6.5. Mor04a, Theorem 6.4.1] Mor04b, Theorem 6.2.1] [Mor12, Corollary 1.25] For any field $k$, the motivic stable homotopy group $\pi_{n, n}^{k}$ is isomorphic to the nth Milnor-Witt $K$-theory group $K_{n}^{M W}(k)$.

Note, in particular, that $\pi_{0,0}^{k}$ is isomorphic to the Grothendieck-Witt ring $G W(k)$ of quadratic forms over $k$. Unlike most of the other results in this article, there is no need for completions in the statement of Theorem 6.5

The element $\epsilon$ that governs graded commutativity corresponds here to $-1-\eta[-1]$, so the last relation of Definition 6.4 is equivalent to the relation $\eta(1-\epsilon)=0$. The element $\rho$ in $\pi_{-1,-1}$ corresponds to [-1], as in Section 2.1.

6.3. The first Milnor-Witt stem. For simplicity let $k$ be a field of characteristic zero. By the work reviewed in Section 6.2 a next logical step is to compute the first Milnor-Witt stem $\Pi_{1}^{k}=\bigoplus_{n \in \mathbb{Z}} \pi_{n+1, n}^{k}$. One of the major inspirations for this calculation is Morel's $\pi_{1}$-conjecture in weight zero, which states that there is a short exact sequence

$$
0 \rightarrow K_{2}^{M}(k) / 24 \rightarrow \pi_{1,0}^{k} \rightarrow k^{\times} / 2 \oplus \mathbb{Z} / 2 \rightarrow 0 .
$$

This conjecture is proved in $\mathrm{RS} \varnothing \mathrm{a}$.

The kernel $K_{2}^{M}(k) / 24$ in (6.1) is generated by the second motivic Hopf map $\nu$ in $\pi_{3,2}^{k}$, in the sense that its elements are all of the form $\alpha \nu$, where $\alpha$ is an element of $\pi_{-2,-2}^{k}$. Such elements $\alpha$ correspond to elements of $K_{2}^{M}(k)$, as in Theorem 6.5.

On the other hand, the image $k^{\times} / 2 \oplus \mathbb{Z} / 2$ has two generators. The second factor is generated by $\eta_{\text {top }}$, i.e., the image of the classical first Hopf map in $\pi_{1,0}^{k}$. The first factor is generated by $\eta \eta_{\text {top }}$, in the sense that its elements are all of the form $\alpha \eta \eta_{\text {top }}$, where $\alpha$ is an element of $\pi_{-1,-1}^{k}$. These generators are subject to the relations $24 \nu=0$ and $12 \nu=\eta^{2} \eta_{\mathrm{top}}$, which are related to the corresponding classical relations $24 \nu=0$ and $12 \nu=\eta^{3}$ in $\pi_{3}$.

It turns out the surjection in (6.1) arises from the unit map for the hermitian $K$-theory spectrum $\mathbf{K Q}$ of quadratic forms. One may speculate that its kernel is the image of a motivic $J$-homomorphism $K_{2}^{M}(k)=\pi_{1,0} G L \rightarrow \pi_{1,0} \mathbf{1}$ for the general linear group GL. The Hopf construction should witness that $\nu$ is in the image of a motivic $J$-homomorphism, so the relation $24 \nu=0$ may be a shadow of some motivic version of the Adams conjecture.

More generally, for every $n \in \mathbb{Z}$, there is an exact sequence RSØa

$$
0 \rightarrow K_{2-n}^{M} / 24(k) \rightarrow \pi_{n+1, n}^{k} \rightarrow \pi_{n+1, n} \mathrm{f}_{0} \mathbf{K Q} .
$$

Here $\mathrm{f}_{0} \mathbf{K Q}$ is the effective slice cover of hermitian $K$-theory (see Section 3). Note that the homotopy groups of $\mathbf{K Q}$ and $\mathrm{f}_{0} \mathbf{K Q}$ agree in nonnegative weight. The 
rightmost map in (6.2) is surjective for $n \geq-4$. In fact, the rightmost map is surjective for all $n$ if $\mathrm{f}_{0} \mathbf{K Q}$ is replaced by the very effective slice cover $\mathbf{k q}=\tilde{\mathrm{f}}_{0} \mathbf{K Q}$ RSØb.

The proof of (6.2) is achieved by performing calculations with the effective slice spectral sequence for the sphere $\mathbf{1}$, converging conditionally to the homotopy of the $\eta$-completion $\mathbf{1}_{\eta}^{\wedge}$. For all integers $n$, there is a canonically induced isomorphism

$$
\pi_{n+1, n} \mathbf{1} \stackrel{\cong}{\longrightarrow} \pi_{n+1, n} \mathbf{1}_{\eta}^{\wedge}
$$

noted in RSØa, Corollary 5.2].

The exact sequence (6.2) vastly generalizes computations in $\mathrm{O} \varnothing 14$ for fields of cohomological dimension at most two, i.e., if Milnor $K$-theory is concentrated in degrees 0,1 , and 2. Examples include algebraically closed fields, finite fields, $p$-adic fields, and totally imaginary number fields (but not $\mathbb{Q}$ or $\mathbb{R}$ ).

It is interesting to compare the above with the computations of unstable motivic homotopy groups of punctured affine spaces in AF15 and AWW17. If $d>3$, the extension for $\pi_{d}\left(\mathbf{A}^{d} \backslash\{0\}\right)$ conjectured by Asok-Fasel [13, Conjecture 7, p. 1894] coincides with (6.2). As noted in AF15, the sheaf version of the exact sequence (6.2) and a conjectural Freudenthal $\mathbb{P}^{1}$-suspension theorem imply Murthy's conjecture on splittings of vector bundles [AF15, Conjecture 1].

6.4. Finite fields. This section describes work of Wilson and Østvær [WØ17] on stable motivic homotopy groups over finite fields of order $q=p^{n}$, where $p$ is an odd prime.

As always, the starting point is the motivic cohomology of a point. Using Example 2.6 and Theorem 2.7, we obtain that $H^{*, *}\left(\mathbb{F}_{q} ; \mathbb{F}_{2}\right)=\mathbb{F}_{2}[u] / u^{2}$.

At this point, the discussion splits into two cases. If $p \equiv 1 \bmod 4$, then -1 is a square in $\mathbb{F}_{q}$, and $\rho$ equals 0 . Consequently, Ext calculations (i.e., motivic Adams $E_{2}$-pages) are essentially the same as in the $\mathbb{C}$-motivic case discussed in Section 2.3 .

On the other hand, if $p \equiv 3 \bmod 4$, then $u$ can be taken to be $\rho$. The Ext groups in this case can be computed with the $\rho$-Bockstein spectral sequence as in Section 2.4 .

However, there are significant differences to the $\mathbb{R}$-motivic case that arise from the relation $\rho^{2}=0$.

In either case, there are interesting motivic Adams differentials for finite fields that have no $\mathbb{C}$-motivic nor $\mathbb{R}$-motivic analogue [WØ17, Corollary 7.12]. More specifically, there are differentials of the form

$$
d_{r}\left(\tau^{k}\right)=u \tau^{k-1} h_{0}^{r}
$$

for some values of $r$ and $k$ that depend on the order $q$ of the finite field $\mathbb{F}_{q}$. These differentials are remarkable because they occur already at the very beginning of the spectral sequence in the 0-stem! The proofs of these differentials depend on a priori knowledge of the motivic cohomology of $\mathbb{F}_{q}$ via its étale cohomology [Sou79, as discussed in Section 7.1

An analysis of the Adams spectral sequence leads to an isomorphism

$$
\pi_{n, 0}^{\mathbb{F}_{q}} \cong \pi_{n}^{s} \oplus \pi_{n+1}^{s} \text {. }
$$

for $0 \leq n \leq 18$. In particular, the groups $\pi_{4,0}^{\mathbb{F}_{q}}$ and $\pi_{12,0}^{\mathbb{F}_{q}}$ are trivial. It is interesting to note that the pattern $\pi_{n, 0}^{\mathbb{F}_{q}} \cong \pi_{n}^{s} \oplus \pi_{n+1}^{s}$ does not hold in general. In fact when 
$q \equiv 5 \bmod 8$ one obtains

$$
\pi_{19,0}^{\mathbb{F}_{q}} \cong(\mathbb{Z} / 8 \oplus \mathbb{Z} / 2) \oplus \mathbb{Z} / 4 \text { and } \pi_{20,0}^{\mathbb{F}_{q}} \cong \mathbb{Z} / 8 \oplus \mathbb{Z} / 2 .
$$

6.5. $\eta$-periodic phenomena. Relatively little is known about $\eta$-periodic phenomena over fields other than $\mathbb{C}$ and $\mathbb{R}$. Sections 4.2 and 5.1 describe the $\eta$-periodic groups $\pi_{*, *}^{\mathbb{C}}\left[\eta^{-1}\right]$ and $\pi_{*, *}^{\mathbb{R}}\left[\eta^{-1}\right]$. Recent work of Wilson Wil18] calculates $\pi_{*, *}^{k}\left[\eta^{-1}\right]$ for $\mathbb{Q}$ and for fields of cohomological dimension at most 2, i.e., for fields whose Milnor $K$-theory vanishes above degree 2. All finite fields satisfy this condition. The $\eta$-periodic groups are described in terms of the Witt group $W(k)$ of quadratic forms over $k$.

For more general fields, the following theorem summarizes what we do know. Recall the notation

$$
\Pi_{m}^{k}=\bigoplus_{n \in \mathbb{Z}} \pi_{m+n, n}^{k}
$$

from Section 5.1

Theorem 6.6. ALP17, Let $k$ be a field such that char $k \neq 2$. Then

$$
\Pi_{m}^{k}\left[\eta^{-1}\right] \otimes \mathbb{Q}=0
$$

for all $m>0$.

Theorem 6.6 leaves open the question of torsion in $\Pi_{m}^{k}\left[\eta^{-1}\right]$. This torsion is likely to be quite interesting.

Additionally, we have some low-dimensional information.

Theorem 6.7. Rön Let $k$ be a field such that char $k \neq 2$. Then $\Pi_{1}^{k}$ and $\Pi_{2}^{k}$ are both zero.

\section{Other MOTIVIC SPECTRA}

One way to obtain information about stable motivic homotopy groups is to consider other motivic spectra that are equipped with unit maps from the motivic sphere spectrum. The homotopy groups of these other motivic spectra can give information about the motivic stable homotopy groups by passing along the unit map. We will discuss a few examples in this section.

Each motivic spectrum discussed below is of fundamental interest in its own right. Their associated cohomology theories detect interesting phenomena in algebraic geometry, but that is not the focus of this discussion. We will not discuss their construction and geometric origins because these topics go beyond the scope of this article.

The unit map for algebraic cobordism factors through $1 / \eta$, so every module over an oriented motivic ring spectrum is $\eta$-complete [RSØ18, Lemma 2.1]. Hence the motivic Eilenberg-Mac Lane spectrum $\mathbf{M} \mathbb{Z}$, the $K$-theory spectrum $\mathbf{K G L}$ and its covers, and the truncated Brown-Peterson spectra $\mathbf{M B P}\langle n\rangle$ are all $\eta$-complete. On the other hand, hermitian $K$-theory $\mathbf{K Q}$ and higher Witt-theory $\mathbf{K W}$ do not coincide with their respective $\eta$-completions. 
7.1. Motivic Eilenberg-Mac Lane spectra. Suppose $\ell$ is prime to the characteristic of the base field $k$. Our first example is the motivic Eilenberg-Mac Lane spectrum $\mathbf{M} \mathbb{Z} / \ell^{\nu}$ that represents motivic cohomology with $\mathbb{Z} / \ell^{\nu}$ coefficients Voe98] [RØ08. The stable homotopy groups of $\mathbf{M} \mathbb{Z} / \ell^{\nu}$ are the same as the cohomology of a point with coefficients in $\mathbb{Z} / \ell^{\nu}$, as described in Section 2.1. The mod- $\ell$ motivic Steenrod algebra is the ring of operations on $\mathbf{M} \mathbb{Z} / \ell$, as described in Section 2.3. This circle of ideas leads eventually to the motivic Adams spectral sequence of Section 2.5.

The Beilinson-Lichtenbaum conjecture, which is a consequence of the Milnor and Bloch-Kato conjectures, offers the following comparison isomorphism due to Voevodsky.

Theorem 7.1. VVoe11] For $p \leq q$ and $X$ a smooth $k$-scheme, the étale sheafification functor induces an isomorphism

$$
H^{p, q}\left(X ; \mathbb{Z} / \ell^{\nu}\right) \stackrel{\cong}{\rightarrow} H_{e ́ t}^{p}\left(X ; \mu_{\ell^{\nu}}^{\otimes q}\right)
$$

between motivic and étale cohomology groups, where $\mu_{\ell^{\nu}}$ is the sheaf of $\ell^{\nu}$ th roots of unity.

This important isomorphism identifies the mod- $\ell^{\nu}$ motivic cohomology of $k$ with the classical cohomology groups of the absolute Galois group of $k$ Ser02].

Let $e$ be the exponent of the multiplicative group $\left(\mathbb{Z} / \ell^{\nu}\right)^{\times}$of units. The sheaf $\mu_{\ell^{\nu}}^{\otimes e}$ is constant, so $H^{0, e}\left(k ; \mathbb{Z} / \ell^{\nu}\right)$ is isomorphic to $H^{0,0}\left(k ; \mathbb{Z} / \ell^{\nu}\right)=\mathbb{Z} / \ell^{\nu}$. Let us choose a generator $\tau_{\ell^{\nu}}$ of $H^{0, e}\left(k ; \mathbb{Z} / \ell^{\nu}\right)$. Using Theorem 7.1, we deduce an isomorphism

$$
H^{p, q}\left(X ; \mathbb{Z} / \ell^{\nu}\right)\left[\tau_{\ell^{\nu}}^{-1}\right] \cong H_{\text {êt }}^{p}\left(X ; \mu_{\ell^{\nu}}^{\otimes q}\right)
$$

for all integers $p, q \in \mathbb{Z}$ and all smooth $k$-schemes $X$. The periodicity discussed here is related to Thomason's seminar paper [Tho85], as well as to a recent generalization to motivic spectra such as algebraic cobordism ELSØ17.

In general, the motivic cohomology groups $H^{p, q}\left(k ; \mathbb{Z} / \ell^{\nu}\right)$ do not have a simple description in terms of Milnor $K$-theory. The case $\nu=1$ is discussed in Section 2.1. If $k$ contains all $\ell^{\nu}$ th roots of unity, then the sheaf $\mu_{\ell^{\nu}}$ is trivial, and Theorem 7.1 gives a practial way of computing $H^{p, q}\left(k ; \mathbb{Z} / \ell^{\nu}\right)$. One way to view these difficulties is that the $\ell$-Bockstein spectral sequence that starts with $H^{*, *}(k ; \mathbb{Z} / \ell)$ and converges to $H^{*, *}\left(k ; \mathbb{Z} / \ell^{\nu}\right)$ is non-trivial.

In addition to $\mathbf{M} \mathbb{Z} / \ell$, we may also consider the motivic Eilenberg-Mac Lane spectrum $\mathbf{M Z}$ that represents integral motivic cohomology. The cofiber sequence

$$
\mathbf{M} \mathbb{Z} \stackrel{\ell^{\nu}}{\longrightarrow} \mathbf{M} \mathbb{Z} \longrightarrow \mathbf{M} \mathbb{Z} / \ell^{\nu}
$$

provides a tool for understanding $\mathbf{M} \mathbb{Z}$ in terms of $\mathbf{M} \mathbb{Z} / \ell^{\nu}$ for each rational prime $\ell$. Its rational part $\mathbf{M} \mathbb{Q}$ identifies with its étale counterpart as in Voe03b, Lemma $6.8]$.

7.2. Algebraic $K$-theory. Our next family of examples arises from $K$-theory. In this section, we will discuss the motivic analogues of the classical periodic $K$-theory spectrum KU and its connective cover ku.

The motivic spectrum KGL represents algebraic $K$-theory Voe98 in the following sense. The homotopy groups $\pi_{n, 0} \mathbf{K G L}$ are isomorphic to the algebraic $K$-theory $K_{n}(k)$ of the base field $k$. In addition, there is a Bott element $\beta$ in $\pi_{2,1} \mathbf{K G L}$, and KGL is periodic in $\beta$. This determines all of the stable homotopy groups of KGL, 
in terms of classical algebraic $K$-theory. The motivic spectrum KGL is analogous to $\mathbf{K U}$ in the classical case.

We next wish to consider the effective slice cover $\mathbf{k g l}=\mathrm{f}_{0} \mathbf{K G L}$, which is analogous to $\mathbf{k u}$ in the classical case. In the case of KGL, the very effective slice cover $\tilde{\mathrm{f}}_{0}$ KGL coincides with $\mathbf{k g l}$ because $\mathbf{k g l}$ is already very effective $\mathbf{S} \varnothing 12$, Corollary 5.13]. In the specific $\mathbb{C}$-motivic case, there is another way to construct the same motivic spectrum $\mathrm{kgl}$ that is more in the spirit of the classical Postnikov tower IS11.

The motivic spectrum $\mathrm{kgl}$ is best understood via its connection to the motivic truncated Brown-Peterson spectrum MBP $\langle 1\rangle$, discussed below in Section 7.3 .

7.3. Motivic Brown-Peterson spectra and truncations. Let MBP denote the motivic Brown-Peterson spectrum at the prime 2 over a characteristic 0 base field $k$ constructed in [HK01] and [Vez01]. This is the universal 2-typical oriented motivic ring spectrum. It turns out that the 2-localized effective slice cover $\mathbf{k g l}_{(2)}$ is the truncated Brown-Peterson spectrum $\mathbf{M B P}\langle 1\rangle$ sitting in a tower

$$
\operatorname{MBP}=\operatorname{MBP}\langle\infty\rangle \rightarrow \cdots \rightarrow \operatorname{MBP}\langle n\rangle \rightarrow \operatorname{MBP}\langle n-1\rangle \rightarrow \cdots \rightarrow \operatorname{MBP}\langle 0\rangle
$$

of MBP-modules. The maps in this tower come from the fact that $\mathbf{M B P}\langle n-1\rangle$ is the cofiber of the map

$$
v_{n}: \operatorname{MBP}\langle n\rangle \rightarrow \operatorname{MBP}\langle n\rangle .
$$

This picture is entirely analogous to the classical situation [JW73], in which $\mathbf{B P}\langle n-$ $1\rangle$ is the cofiber of

$$
\mathbf{B P}\langle n\rangle \rightarrow \mathbf{B P}\langle n\rangle .
$$

The motivic spectrum $\mathbf{M B P}\langle 0\rangle=\mathbf{M} \mathbb{Z}_{(2)}$ is the 2-local motivic Eilenberg-Mac Lane spectrum by the theorem of Hopkins, Morel, and Hoyois Hoy15. (At odd primes, $\mathbf{M B P}\langle 0\rangle$ is an Adams summand of localized connective algebraic $K$-theory [NSØ15, §4].) For $n>1$ we can view the groups $\pi_{*} \mathbf{M B P}\langle n\rangle$ as higher height generalizations of the algebraic $K$-theory groups of the base field.

In order to understand the homology of $\mathbf{M B P}\langle n\rangle$ as a comodule over $A_{*}$ we employ the auxiliary Hopf algebroids

$$
\begin{aligned}
\mathcal{E}(n)_{*} & =\left(\mathbb{M}_{2}, A_{*} /\left(\xi_{1}, \xi_{2}, \ldots\right)+\left(\tau_{n+1}, \tau_{n+2}, \ldots\right)\right) \\
& =\left(\mathbb{M}_{2}, \mathbb{M}_{2}\left[\tau_{0}, \ldots, \tau_{n}\right] /\left(\tau_{i}^{2}-\rho \tau_{i+1}, \tau_{n}^{2}\right)\right) .
\end{aligned}
$$

We permit $n=\infty$, in which case

$$
\begin{aligned}
\mathcal{E}(\infty)_{*} & =\left(\mathbb{M}_{2}, A_{*} /\left(\xi_{1}, \xi_{2}, \ldots\right)\right) \\
& =\left(\mathbb{M}_{2}, \mathbb{M}_{2}\left[\tau_{0}, \tau_{1}, \ldots\right] /\left(\tau_{i}^{2}-\rho \tau_{i+1}\right)\right) .
\end{aligned}
$$

By Orm11 there is an isomorphism of Hopf algebroids

$$
\mathbf{M} Z / 2_{*} \operatorname{MBP}\langle n\rangle \cong A_{*} \square_{\mathcal{E}(n)_{*}} \mathbb{M}_{2} .
$$

By a standard change-of-rings isomorphism, the Adams $E_{2}$-page for $\mathbf{M B P}\langle n\rangle$ identifies with

$$
\operatorname{Ext}_{\mathcal{E}(n)}\left(\mathbb{M}_{2}, \mathbb{M}_{2}\right) \text {. }
$$

For explicit calculations of $\pi_{*} \operatorname{MBP}\langle n\rangle$ over $\mathbb{C}, \mathbb{R}, \mathbb{Q}_{p}$, and $\mathbb{Q}$ we refer to [ØØ13]. 
7.4. Hermitian $K$-theory. In this section, we consider the motivic versions of the classical real $K$-theory spectrum $\mathbf{K O}$ and its connective cover ko.

The motivic spectrum KQ represents Karoubi's hermitian $K$-theory Hor05. Its effective slice cover $f_{0} \mathbf{K Q}$ does not coincide with its very effective slice cover $\mathbf{k q}:=\tilde{\mathrm{f}}_{0} \mathbf{K Q}$ studied in [ARØ17. We note that $\mathbf{k q}$ and $\mathbf{k g l}$ are connected via the motivic Hopf map $\eta: \mathbb{A}^{2} \backslash\{0\} \rightarrow \mathbb{P}^{1}$ in the cofiber sequence

$$
\Sigma^{1,1} \mathbf{k q} \stackrel{\eta}{\rightarrow} \mathbf{k q} \rightarrow \mathbf{k g l} .
$$

An analogous cofiber sequence for the effective slice covers of KQ and KGL does not exist by the proof of $\mathrm{RS} \varnothing 18$, Corollary 5.1]. By (7.3) the Betti realization of kq identifies with ko and one can calculate the mod-2 motivic homology $\mathbf{M} \mathbb{Z} / 2_{*}$ kq as

$$
A_{*} \square_{A(1) *} \mathbb{M}_{2},
$$

where $A(1)_{*}$ is the Hopf algebroid

$$
\begin{aligned}
A(1)_{*} & =\left(\mathbb{M}_{2}, A_{*} /\left(\xi_{1}^{2}, \xi_{2}, \xi_{3} \ldots\right)+\left(\tau_{2}, \tau_{3}, \ldots\right)\right) \\
& =\left(\mathbb{M}_{2}, \mathbb{M}_{2}\left[\tau_{0}, \tau_{1}, \xi_{1}\right] /\left(\tau_{0}^{2}+\tau \xi_{1}+\rho \tau_{1}+\rho \tau_{0} \xi_{1}, \xi_{1}^{2}, \tau_{1}^{2}\right)\right.
\end{aligned}
$$

By change-of-rings, the Adams $E_{2}$-page for kq takes the form

$$
\operatorname{Ext}_{A(1)}^{*, *}\left(\mathbf{M}_{2}, \mathbf{M}_{2}\right) \text {. }
$$

As usual, this Adams spectral sequence computes the homotopy groups of kq completed at 2 and $\eta$. For explicit calculations with (7.4) over $\mathbb{C}$ and $\mathbb{R}$, we refer to IS11] and Hil11.

7.5. Higher Witt theory. In this section we review the proof of Milnor's conjecture on quadratic forms based on the effective slice spectral sequence for higher Witt-theory $\mathbf{K W}$ [RØ16]. Recall that $\mathbf{K W}$ is defined by inverting $\eta$ on $\mathbf{K Q}$.

Suppose $k$ is a field of characteristic unequal to 2. Recall the Milnor $K$-theory of $k$ described in Section 2.1. In degrees zero, one, and two, these groups agree with Quillen's $K$-groups, but for higher degrees they differ in general. Milnor Mil69] proposed two conjectures relating $K_{*}^{M}(k) / 2$ to the mod-2 Galois cohomology ring $H^{*}\left(F ; \mu_{2}\right)$ and the graded Witt ring $G r W_{*}^{I}(k)=\oplus_{q>0} I(k)^{q} / I(k)^{q+1}$ for the fundamental ideal $I(k)$ of even dimensional forms in the Witt ring $W(k)$, in the form of two graded ring homomorphisms:

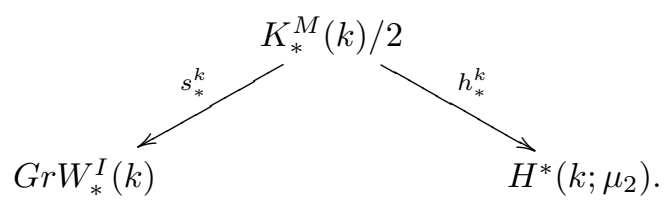

The Milnor conjecture on Galois cohomology states that $h_{*}^{k}$ is an isomorphism, while the Milnor conjecture on quadratic forms states that $s_{*}^{k}$ is an isomorphism. The proofs of both conjectures Voe03b OVV07 are two striking applications of motivic homotopy theory.

The slices of $\mathbf{K W}$ were described in Part (3) of Theorem 3.4. We record the first differentials in the effective slice spectral sequence for $\mathbf{K W}$ as maps between motivic spectra. The differential

$$
d_{1}^{\mathbf{K W}}(q): \mathbf{s}_{q} \mathbf{K W} \rightarrow \Sigma^{1,0} \mathbf{s}_{q+1} \mathbf{K W}
$$


is a map of the form

$$
\bigvee_{i \in \mathbb{Z}} \Sigma^{2 i+q, q} \mathbf{M} \mathbb{Z} / 2 \rightarrow \Sigma^{2,1} \bigvee_{j \in \mathbb{Z}} \Sigma^{2 j+q, q} \mathbf{M} \mathbb{Z} / 2
$$

Let $d_{1}^{\mathbf{K W}}(q, i)$ denote the restriction of $d_{1}^{\mathbf{K W}}(q)$ to the $i$ th summand $\Sigma^{2 i+q, q} \mathbf{M} \mathbb{Z} / 2$ of $\mathbf{s}_{q} \mathbf{K W}$. By comparing with motivic cohomology operations of weight one, it suffices to consider $d_{1}^{\mathbf{K W}}(q, i)$ as a map from $\Sigma^{2 i+q, q} \mathbf{M} \mathbb{Z} / 2$ to

$$
\Sigma^{2 i+q+4, q+1} \mathbf{M} \mathbb{Z} / 2 \vee \Sigma^{2 i+q+2, q+1} \mathbf{M} \mathbb{Z} / 2 \vee \Sigma^{2 i+q, q+1} \mathbf{M} \mathbb{Z} / 2
$$

The latter map affords the closed formula

$$
d_{1}^{\mathbf{K W}}(q, i)= \begin{cases}\left(\mathrm{Sq}^{3} \mathrm{Sq}^{1}, \mathrm{Sq}^{2}, 0\right) & i-2 q \equiv 0 \bmod 4 \\ \left(\mathrm{Sq}^{3} \mathrm{Sq}^{1}, \mathrm{Sq}^{2}+\rho \mathrm{Sq}^{1}, \tau\right) & i-2 q \equiv 2 \bmod 4\end{cases}
$$

This sets the stage for the proof by Röndigs and the second author [RØ16] of Milnor's conjecture on quadratic forms formulated in [Mil69, Question 4.3]. For fields of characteristic zero this conjecture was first shown by Orlov, Vishik and Voevodsky in OVV07, and by Morel Mor99a using different approaches.

According to Part (3) of Theorem 3.4, the effective slice spectral sequence for KW fills out the entire upper half-plane. A strenuous computation using (7.6), Adem relations, and the action of the Steenrod squares on the mod-2 motivic cohomology ring of $k$ shows that it collapses. A key point from Theorem 2.7 is that if $0 \leq p \leq q$ and $a$ belongs to $H^{p, q}\left(k ; \mathbb{F}_{2}\right)$, then $a$ equals $\tau^{q-p} c$ for some $c$ in $H^{p, p}\left(k ; \mathbb{F}_{2}\right)$. The action of the Steenrod operations in weight at most 1 is then completely described by the formulas

$$
\begin{gathered}
\mathrm{Sq}^{1}\left(\tau^{n} c\right)= \begin{cases}\rho \tau^{n-1} c & n \equiv 1 \bmod 2 \\
0 & n \equiv 0 \bmod 2\end{cases} \\
\mathrm{Sq}^{2}\left(\tau^{n} c\right)= \begin{cases}\rho^{2} \tau^{n-1} c & n \equiv 2,3 \bmod 4 \\
0 & n \equiv 0,1 \bmod 4\end{cases}
\end{gathered}
$$

With the above in hand, a long calculation shows that the effective slice $E_{2}$-page for $\mathbf{K W}$ in degree $(p, q)$ equals $H^{p, p}\left(k ; \mathbb{F}_{2}\right)$ if $p \bmod 4$, and equals zero otherwise.]

To connect this computation with the theory of quadratic forms, one shows the spectral sequence converges to the filtration of the Witt ring $W(k)$ by the powers of the fundamental ideal $I(k)$ of even dimensional forms. By identifying motivic cohomology with Galois cohomology for fields we arrive at the following result.

Theorem 7.2. $\mathrm{R} \varnothing 16$, Theorem 1.1] When $\operatorname{char}(k) \neq 2$, the effective slice spectral sequence for $\mathbf{K W}$ converges and furnishes a complete set of invariants

$$
I(k)^{q} / I(k)^{q+1} \cong H^{q}\left(k ; \mu_{2}\right)
$$

for quadratic forms over $k$ with values in the mod-2 Galois cohomology ring.

\section{Future Directions}

The purpose of this section is to encourage further research into motivic stable homotopy groups by describing some specific projects. 
Problem 8.1. Classical periodicity consists of existence results, such as the existence of Morava $K$-theories and the existence of $v_{n}$-self maps; and uniqueness results, such as the fact that a finite complex possesses a unique $v_{n}$-self map (at least up to taking powers). In the $\mathbb{C}$-motivic situation, many of the analogous existence results have been established.

On the other hand, much work remains to be done on the uniqueness results. In particular, it is not known whether there are any periodicities in addition to those discovered by Andrews [And18, Gheorghe Ghe17, and Krause [Kra18. Also, it turns out that a finite complex can possess more than one type of periodic self-map. It is not yet understood how periodicities of different types can co-exist, nor what that means for the structure of $\mathbb{C}$-motivic stable homotopy groups.

To date, there is little work on exotic nilpotence and periodicity over other base fields. At least some of the same phenomena must occur. See [Joa15] and Hor18] for some first results.

Problem 8.2. Currently available techniques allow for many more $\mathbb{R}$-motivic computations than have so far been carried out. Preliminary data suggests many interesting connections to other aspects of stable homotopy theory. In particular, it appears that the $\mathbb{R}$-motivic image of $J$ has order greater than the classical image of $J$ [BI]. A full accounting of this phenomenon is needed. Both the motivic Adams spectral sequence and the slice spectral sequence ought to be important tools.

The $\mathbb{R}$-motivic stable homotopy category $\mathbf{S H}(\mathbb{R})$ appears to be closely related to the $C_{2}$-equivariant stable homotopy category. Data suggests that the $C_{2}$-equivariant stable homotopy category is the same as " $\tau$-periodic $\mathbb{R}$-motivic stable homotopy theory".

Recent on-going work of Behrens and Shah addresses this issue.

Bruner and Greenlees BG95 showed how to reformulate the classical Mahowald invariant Beh07 in terms of $C_{2}$-equivariant stable homotopy groups. The $\mathbb{R}$ motivic stable homotopy groups are somewhat easier to study than the $C_{2}$-equivariant stable homotopy groups, and they also ought to be useful for Mahowald invariants.

Problem 8.3. In classical homotopy, the topological modular forms spectrum tmf is an essential tool for studying stable homotopy groups. The cohomology of $\operatorname{tmf}$ is $A / / A(2)$, where $A$ is the classical Steenrod algebra and $A(2)$ is the subalgebra of $A$ generated by $\mathrm{Sq}^{1}, \mathrm{Sq}^{2}$, and $\mathrm{Sq}^{4}$. This means that the homotopy groups of $\operatorname{tmf}$ can be computed by an Adams spectral sequence whose $E(2)$-page is the cohomology of $A(2)$. The Adams spectral sequence for tmf can be completely analyzed, and this analysis provides much information about the classical stable homotopy groups.

It is likely that a similar story plays out in the motivic situation. We would like to know that a "motivic modular forms" spectrum mmf exists over an arbitrary field $k$. The motivic stable homotopy groups of $m m f$ ought to be computable by an Adams spectral sequence whose $E_{2}$-page is the cohomology of the subalgebra $A(2)$ of the $k$-motivic Steenrod algebra $A$.

Problem 8.4. Formula (3.7) describes the $E_{1}$-page of the effective slice spectral sequence, in terms of the classical Adams-Novikov $E_{2}$-page and in terms of motivic cohomology with coefficients in $\mathbb{Z}$ and $\mathbb{Z} / 2^{n}$. Preliminary calculations indicate that this $E_{1}$-page has interesting "exotic" products that are not simply seen by the 
classical Adams-Novikov $E_{2}$-page. This product structure deserves careful study in low dimensions. See Remark 3.3 for more discussion.

Problem 8.5. The $E_{1}$-page of the effective slice spectral sequence can be described over a general base field $k$, at least in terms of the Milnor $K$-theory of $k$. Some differentials have been understood in general in very low dimensions [RSØa. These results about effective slice differentials ought to be accessible in a larger range of dimensions, at least relative to arithmetic input from $k$.

Problem 8.6. Section 7.5 discusses how the effective slice filtration for $\mathbf{K W}$ informs us about quadratic forms over a field $k$. It is possible that these ideas can be extended to study quadratic forms over smooth $k$-schemes.

\section{REFERENCES}

[Ada66] J. F. Adams, On the groups $J(X)$. IV, Topology 5 (1966), 21-71. MR0198470 (33 \#6628)

[Ada74] _ Stable homotopy and generalised homology, University of Chicago Press, Chicago, Ill.-London, 1974. Chicago Lectures in Mathematics. MR0402720

[ALP17] A. Ananyevskiy, M. Levine, and I. Panin, Witt sheaves and the $\eta$-inverted sphere spectrum, J. Topol. 10 (2017), no. 2, 370-385, DOI 10.1112/topo.12015. MR3653315

[ARØ17] A. Ananyevskiy, O. Röndigs, and P. A. Østvær, On very effective hermitian K-theory (2017), preprint, available at arXiv:1712.01349

[And18] M. J. Andrews, New families in the homotopy of the motivic sphere spectrum, Proc. Amer. Math. Soc. 146 (2018), no. 6, 2711-2722, DOI 10.1090/proc/13940. MR3778171

[AM17] M. Andrews and H. Miller, Inverting the Hopf map, J. Topol. 10 (2017), no. 4, 11451168, DOI 10.1112/topo.12034. MR3743072

[AF15] A. Asok and J. Fasel, Splitting vector bundles outside the stable range and $\mathbb{A}^{1}$ homotopy sheaves of punctured affine spaces, J. Amer. Math. Soc. 28 (2015), no. 4, 1031-1062, DOI 10.1090/S0894-0347-2014-00818-3. MR3369908

[AWW17] A. Asok, K. Wickelgren, and B. Williams, The simplicial suspension sequence in $\mathbb{A}^{1}$ homotopy, Geom. Topol. 21 (2017), no. 4, 2093-2160, DOI 10.2140/gt.2017.21.2093. MR3654105

[AH61] M. F. Atiyah and F. Hirzebruch, Vector bundles and homogeneous spaces, Proc. Sympos. Pure Math., Vol. III, American Mathematical Society, Providence, R.I., 1961, pp. 7-38. MR0139181

[Bac18] T. Bachmann, Motivic and real étale stable homotopy theory, Compos. Math. 154 (2018), no. 5, 883-917, DOI 10.1112/S0010437X17007710. MR3781990

[BMT70] M. G. Barratt, M. E. Mahowald, and M. C. Tangora, Some differentials in the Adams spectral sequence. II, Topology 9 (1970), 309-316. MR0266215 (42 \#1122)

[Beh07] M. Behrens, Some root invariants at the prime 2, Proceedings of the Nishida Fest (Kinosaki 2003), Geom. Topol. Monogr., vol. 10, Geom. Topol. Publ., Coventry, 2007, pp. 1-40, DOI 10.2140/gtm.2007.10.1. MR2402775

[BI] E. Belmont and D. C. Isaksen, The $\mathbb{R}$-motivic Adams spectral sequence, in preparation.

[Boa99] J. M. Boardman, Conditionally convergent spectral sequences, Homotopy invariant algebraic structures (Baltimore, MD, 1998), Contemp. Math., vol. 239, Amer. Math. Soc., Providence, RI, 1999, pp. 49-84, DOI 10.1090/conm/239/03597. MR1718076

[Bru84] R. Bruner, A new differential in the Adams spectral sequence, Topology 23 (1984), no. 3, 271-276, DOI 10.1016/0040-9383(84)90010-7. MR770563 (86c:55016)

[Bru89] R. R. Bruner, Calculation of large Ext modules, Computers in geometry and topology (Chicago, IL, 1986), Lecture Notes in Pure and Appl. Math., vol. 114, Dekker, New York, 1989, pp. 79-104. MR988692

[Bru93] _ Ext in the nineties, Algebraic topology (Oaxtepec, 1991), Contemp. Math., vol. 146, Amer. Math. Soc., Providence, RI, 1993, pp. 71-90. MR1224908

[Bru97] — The cohomology of the mod 2 Steenrod algebra: A computer calculation 37 (1997).

[BG95] R. Bruner and J. Greenlees, The Bredon-Löffler conjecture, Experiment. Math. 4 (1995), no. 4, 289-297. MR1387694 
[DFHH14] C. L. Douglas, J. Francis, A. G. Henriques, and M. A. Hill (eds.), Topological modular forms, Mathematical Surveys and Monographs, vol. 201, American Mathematical Society, Providence, RI, 2014. MR3223024

[DK18] A. Druzhinin and J. I. Kylling, Framed correspondences and the zeroth stable motivic homotopy group in odd characteristic (2018), preprint, available at arXiv:1809.03238.

[Dug14] D. Dugger, Coherence for invertible objects and multigraded homotopy rings, Algebr. Geom. Topol. 14 (2014), no. 2, 1055-1106, DOI 10.2140/agt.2014.14.1055. MR3180827

[DI10] D. Dugger and D. C. Isaksen, The motivic Adams spectral sequence, Geom. Topol. 14 (2010), no. 2, 967-1014, DOI 10.2140/gt.2010.14.967. MR2629898 (2011e:55024)

[DI13] Motivic Hopf elements and relations, New York J. Math. 19 (2013), 823-871. MR3141814

[DI17] _ Low-dimensional Milnor-Witt stems over $\mathbb{R}$, Ann. K-Theory 2 (2017), no. 2, 175-210. MR3590344

[ELSØ17] E. Elmanto, M. Levine, M. Spitzweck, and P. A. Østvær, Motivic Landweber exact theories and étale cohomology (2017), preprint, available at arXiv:1711.06258

[Ghe17] B. Gheorghe, Exotic motivic periodicities (2017), preprint, available at arXiv:1709.00915.

[Ghe] , The motivic cofiber of $\tau$, Doc. Math., to appear.

[GWX18] B. Gheorghe, G. Wang, and Z. Xu, The special fiber of the motivic deformation of the stable homotopy category is algebraic (2018), preprint, available at arXiv:1809.09290.

[GI15] B. J. Guillou and D. C. Isaksen, The $\eta$-local motivic sphere, J. Pure Appl. Algebra 219 (2015), no. 10, 4728-4756, DOI 10.1016/j.jpaa.2015.03.004. MR3346515

[GI16] $\longrightarrow$ The $\eta$-inverted $\mathbb{R}$-motivic sphere, Algebr. Geom. Topol. 16 (2016), no. 5, 3005-3027. MR3572357

[GRSØ12] J. J. Gutiérrez, O. Röndigs, M. Spitzweck, and P. A. Østvær, Motivic slices and coloured operads, J. Topol. 5 (2012), no. 3, 727-755, DOI 10.1112/jtopol/jts015. MR2971612

[Hil11] M. A. Hill, Ext and the motivic Steenrod algebra over $\mathbb{R}$, J. Pure Appl. Algebra 215 (2011), no. 5, 715-727, DOI 10.1016/j.jpaa.2010.06.017. MR2747214 (2012i:55020)

[HHR16] M. A. Hill, M. J. Hopkins, and D. C. Ravenel, On the nonexistence of elements of Kervaire invariant one, Ann. of Math. (2) 184 (2016), no. 1, 1-262, DOI 10.4007/annals.2016.184.1.1. MR3505179

[Hor05] J. Hornbostel, $A^{1}$-representability of Hermitian K-theory and Witt groups, Topology 44 (2005), no. 3,661-687, DOI 10.1016/j.top.2004.10.004. MR2122220

[Hor18] _ Some comments on motivic nilpotence, Trans. Amer. Math. Soc. 370 (2018), no. 4, 3001-3015, DOI 10.1090/tran/7324. With an appendix by Marcus Zibrowius. MR3748592

[Hoy15] M. Hoyois, From algebraic cobordism to motivic cohomology, J. Reine Angew. Math. 702 (2015), 173-226, DOI 10.1515/crelle-2013-0038. MR3341470

[HKØ17] M. Hoyois, S. Kelly, and P. A. Østvær, The motivic Steenrod algebra in positive characteristic, J. Eur. Math. Soc. (JEMS) 19 (2017), no. 12, 3813-3849, DOI 10.4171/JEMS/754. MR3730515

[HK01] P. Hu and I. Kriz, Some remarks on Real and algebraic cobordism, K-Theory 22 (2001), no. 4, 335-366, DOI 10.1023/A:1011196901303. MR1847399

[HKO11a] P. Hu, I. Kriz, and K. Ormsby, Remarks on motivic homotopy theory over algebraically closed fields, J. K-Theory 7 (2011), no. 1, 55-89, DOI 10.1017/is010001012jkt098. MR2774158 (2012b:14040)

[HKO11b] - Convergence of the motivic Adams spectral sequence, J. K-Theory 7 (2011), no. 3, 573-596, DOI 10.1017/is011003012jkt150. MR2811716

[Isa] D. C. Isaksen, Stable stems, Mem. Amer. Math. Soc., to appear.

[Isa14] —, Classical and motivic Adams charts (2014), preprint, available at arXiv: 1401.4983

[IS11] D. C. Isaksen and A. Shkembi, Motivic connective K-theories and the cohomology of A(1), J. K-Theory 7 (2011), no. 3, 619-661, DOI 10.1017/is011004009jkt154. MR2811718 (2012h:19012)

[IWX] D. C. Isaksen, G. Wang, and Z. Xu, More stable stems, in preparation.

[IX15] D. C. Isaksen and Z. Xu, Motivic stable homotopy and the stable 51 and 52 stems, Topology Appl. 190 (2015), 31-34, DOI 10.1016/j.topol.2015.04.008. MR3349503 
[Jar00] J. F. Jardine, Motivic symmetric spectra, Doc. Math. 5 (2000), 445-552. MR1787949

[Joa15] R. Joachimi, Thick ideals in equivariant and motivic stable homotopy categories (2015), Ph.D. thesis.

[JW73] D. C. Johnson and W. S. Wilson, Projective dimension and Brown-Peterson homology, Topology 12 (1973), 327-353, DOI 10.1016/0040-9383(73)90027-X. MR0334257

[Kra18] A. Krause, Periodicity in motivic homotopy theory and over $B P_{*} B P(2018)$, Ph.D. thesis.

[KW18] J. I. Kylling and G. M. Wilson, Strong convergence in the motivic Adams spectral sequence (2018), preprint.

[Lev08] M. Levine, The homotopy coniveau tower, J. Topol. 1 (2008), no. 1, 217-267, DOI 10.1112/jtopol/jtm004. MR2365658

[Lev14] _ A comparison of motivic and classical stable homotopy theories, J. Topol. 7 (2014), no. 2, 327-362, DOI 10.1112/jtopol/jtt031. MR3217623

[Mag02] B. A. Magurn, An algebraic introduction to K-theory, Encyclopedia of Mathematics and its Applications, vol. 87, Cambridge University Press, Cambridge, 2002. MR1906572

[MT67] M. Mahowald and M. Tangora, Some differentials in the Adams spectral sequence, Topology 6 (1967), 349-369. MR0214072 (35 \#4924)

[Man18] L. Mantovani, Localizations and completions in motivic homotopy theory (2018), preprint, available at arXiv:1810.04134

[May65] J. P. May, The cohomology of restricted Lie algebras and of Hopf algebras, Bull. Amer. Math. Soc. 71 (1965), 372-377, DOI 10.1090/S0002-9904-1965-11300-3. MR0185595

[13] Algebraic K-theory and motivic cohomology, Oberwolfach Rep. 10 (2013), no. 2, 1861-1913, DOI 10.4171/OWR/2013/32. Abstracts from the workshop held June 2329, 2013; Organized by T. Geisser, A. Huber-Klawitter, U. Jannsen and M. Levine. MR3013936

[Mil75] H. R. Miller, Some algebraic aspects of the Adams-Novikov spectral sequence, ProQuest LLC, Ann Arbor, MI, 1975. Thesis (Ph.D.)-Princeton University. MR2625232

[Mil60] J. Milnor, On the cobordism ring $\Omega^{*}$ and a complex analogue. I, Amer. J. Math. 82 (1960), 505-521, DOI 10.2307/2372970. MR0119209

[Mil69] _ Algebraic K-theory and quadratic forms, Invent. Math. 9 (1969/1970), 318344. MR0260844

[Mor99a] F. Morel, Suite spectrale d'Adams et invariants cohomologiques des formes quadratiques, C. R. Acad. Sci. Paris Sér. I Math. 328 (1999), no. 11, 963-968, DOI 10.1016/S0764-4442(99)80306-1 (French, with English and French summaries). MR1696188 (2000d:11056)

[Mor99b] - Théorie homotopique des schémas, Astérisque 256 (1999), vi+119 (French, with English and French summaries). MR1693330 (2000j:14031)

[Mor04a] _ An introduction to $\mathbb{A}^{1}$-homotopy theory, Contemporary developments in algebraic $K$-theory, ICTP Lect. Notes, XV, Abdus Salam Int. Cent. Theoret. Phys., Trieste, 2004, pp. 357-441 (electronic). MR2175638 (2006m:19007)

[Mor04b] _ On the motivic $\pi_{0}$ of the sphere spectrum, Axiomatic, enriched and motivic homotopy theory, NATO Sci. Ser. II Math. Phys. Chem., vol. 131, Kluwer Acad. Publ., Dordrecht, 2004, pp. 219-260, DOI 10.1007/978-94-007-0948-5_7. MR2061856

[Mor06] _ Rationalized motivic sphere spectrum and rational motivic cohomology (2006), preprint.

[Mor12], $\mathbb{A}^{1}$-algebraic topology over a field, Lecture Notes in Mathematics, vol. 2052, Springer, Heidelberg, 2012. MR2934577

[MV99] F. Morel and V. Voevodsky, $\mathbf{A}^{1}$-homotopy theory of schemes, Inst. Hautes Études Sci. Publ. Math. 90 (1999), 45-143 (2001). MR1813224 (2002f:14029)

[Mos70] R. M. F. Moss, Secondary compositions and the Adams spectral sequence, Math. Z. 115 (1970), 283-310. MR0266216 (42 \#1123)

[Nas] C. Nassau, http://www.nullhomotopie.de/

[NSØ09] N. Naumann, M. Spitzweck, and P. A. Østvær, Motivic Landweber exactness, Doc. Math. 14 (2009), 551-593. MR2565902

[NSØ15] — Existence and uniqueness of $E_{\infty}$ structures on motivic $K$-theory spectra, J. Homotopy Relat. Struct. 10 (2015), no. 3, 333-346, DOI 10.1007/s40062-013-0062-3. MR3385689 
[Nes18] A. Neshitov, Framed correspondences and the Milnor-Witt K-theory, J. Inst. Math. Jussieu 17 (2018), no. 4, 823-852, DOI 10.1017/S1474748016000190. MR3835524

[Nov67] S. P. Novikov, Methods of algebraic topology from the point of view of cobordism theory, Izv. Akad. Nauk SSSR Ser. Mat. 31 (1967), 855-951 (Russian). MR0221509

[OVV07] D. Orlov, A. Vishik, and V. Voevodsky, An exact sequence for $K_{*}^{M} / 2$ with applications to quadratic forms, Ann. of Math. (2) 165 (2007), no. 1, 1-13, DOI 10.4007/annals.2007.165.1. MR2276765

[Orm11] K. M. Ormsby, Motivic invariants of p-adic fields, J. K-Theory 7 (2011), no. 3, 597618, DOI 10.1017/is011004017jkt153. MR2811717

[OØ13] K. M. Ormsby and P. A. Østvær, Motivic Brown-Peterson invariants of the rationals, Geom. Topol. 17 (2013), no. 3, 1671-1706, DOI 10.2140/gt.2013.17.1671. MR3073932

[OØ14] Stable motivic $\pi_{1}$ of low-dimensional fields, Adv. Math. 265 (2014), 97-131, DOI 10.1016/j.aim.2014.07.024. MR3255457

[Pst18] P. Pstragowski, Synthetic spectra and the cellular motivic category (2018), preprint, available at arXiv:1803.01804

[Rav86] D. C. Ravenel, Complex cobordism and stable homotopy groups of spheres, Pure and Applied Mathematics, vol. 121, Academic Press, Inc., Orlando, FL, 1986. MR860042 (87j:55003)

[Rön] O. Röndigs, On the $\eta$-inverted sphere, Tata Institute of Fundamental Research Publications 19, to appear.

[RØ08] O. Röndigs and P. A. Østvær, Modules over motivic cohomology, Adv. Math. 219 (2008), no. 2, 689-727, DOI 10.1016/j.aim.2008.05.013. MR2435654

[RØ16] O. Röndigs and P. A. Østvær, Slices of hermitian K-theory and Milnor's conjecture on quadratic forms, Geom. Topol. 20 (2016), no. 2, 1157-1212, DOI 10.2140/gt.2016.20.1157. MR3493102

[RSØ18] O. Röndigs, M. Spitzweck, and P. A. Østvær, The motivic Hopf map solves the homotopy limit problem for K-theory, Doc. Math. 23 (2018), 1405-1424.

[RSØa] — The first stable homotopy groups of motivic spheres, Ann. of Math., to appear.

[RSØb] - The second stable homotopy groups of motivic spheres, in preparation.

[Ser02] J.-P. Serre, Galois cohomology, Corrected reprint of the 1997 English edition, Springer Monographs in Mathematics, Springer-Verlag, Berlin, 2002. Translated from the French by Patrick Ion and revised by the author. MR1867431

[Sou79] C. Soulé, $K$-théorie des anneaux d'entiers de corps de nombres et cohomologie étale, Invent. Math. 55 (1979), no. 3, 251-295, DOI 10.1007/BF01406843 (French). MR553999

[Spi10] M. Spitzweck, Relations between slices and quotients of the algebraic cobordism spectrum, Homology Homotopy Appl. 12 (2010), no. 2, 335-351. MR2771593

[Spi12] — A commutative $\mathbb{P}^{1}$-spectrum representing motivic cohomology over Dedekind domains (2012), preprint, available at arXiv:1207.4078

[Spi14] _ Algebraic cobordism in mixed characteristic (2014), preprint, available at arXiv:1404.2542

[SØ12] M. Spitzweck and P. A. Østvær, Motivic twisted K-theory, Algebr. Geom. Topol. 12 (2012), no. 1, 565-599, DOI 10.2140/agt.2012.12.565. MR2916287

[Sta16] S.-T. Stahn, The motivic Adams-Novikov spectral sequence at odd primes over $\mathbb{C}$ and $\mathbb{R}$ (2016), preprint, available at arXiv:1606.06085.

[Tho85] R. W. Thomason, Algebraic K-theory and étale cohomology, Ann. Sci. École Norm. Sup. (4) 18 (1985), no. 3, 437-552. MR826102

[Tho16] R. Thornton, The homogeneous spectrum of Milnor-Witt K-theory, J. Algebra 459 (2016), 376-388, DOI 10.1016/j.jalgebra.2016.04.012. MR3503978

[Vez01] G. Vezzosi, Brown-Peterson spectra in stable $\mathbb{A}^{1}$-homotopy theory, Rend. Sem. Mat. Univ. Padova 106 (2001), 47-64. MR1876212

[Voe98] V. Voevodsky, $\mathbb{A}^{1}$-homotopy theory, Proceedings of the International Congress of Mathematicians, Vol. I (Berlin, 1998), 1998, pp. 579-604 (electronic). MR1648048 (99j:14018)

[Voe02a] _ Open problems in the motivic stable homotopy theory. I, Motives, polylogarithms and Hodge theory, Part I (Irvine, CA, 1998), Int. Press Lect. Ser., vol. 3, Int. Press, Somerville, MA, 2002, pp. 3-34. MR1977582 
[Voe02b] — A possible new approach to the motivic spectral sequence for algebraic $K$-theory, Recent progress in homotopy theory (Baltimore, MD, 2000), Contemp. Math., vol. 293, Amer. Math. Soc., Providence, RI, 2002, pp. 371-379, DOI 10.1090/conm/293/04956. MR1890744

[Voe03a] _ Reduced power operations in motivic cohomology, Publ. Math. Inst. Hautes Études Sci. 98 (2003), 1-57, DOI 10.1007/s10240-003-0009-z. MR2031198 (2005b:14038a)

[Voe03b] _ Motivic cohomology with $\mathbf{Z} / 2$-coefficients, Publ. Math. Inst. Hautes Études Sci. 98 (2003), 59-104, DOI 10.1007/s10240-003-0010-6. MR2031199 (2005b:14038b)

[Voe11] _ On motivic cohomology with $\mathbf{Z} /$ l-coefficients, Ann. of Math. (2) 174 (2011), no. 1, 401-438. MR2811603

[WX] G. Wang and Z. Xu, On the uniqueness of the smooth structure of the 61-sphere, Ann. of Math., to appear.

[Wei13] C. A. Weibel, The $K$-book, Graduate Studies in Mathematics, vol. 145, American Mathematical Society, Providence, RI, 2013. An introduction to algebraic $K$-theory. MR3076731

[Wil18] G. M. Wilson, The eta-inverted sphere over the rationals, Algebr. Geom. Topol. 18 (2018), no. 3, 1857-1881, DOI 10.2140/agt.2018.18.1857. MR3784021

[WØ17] G. M. Wilson and P. A. Østvær, Two-complete stable motivic stems over finite fields, Algebr. Geom. Topol. 17 (2017), no. 2, 1059-1104. MR3623682

\section{REFERENCES}

[Ada66] J. F. Adams, On the groups $J(X)$. IV, Topology 5 (1966), 21-71. MR0198470 (33 \#6628)

[Ada74] - Stable homotopy and generalised homology, University of Chicago Press, Chicago, Ill.-London, 1974. Chicago Lectures in Mathematics. MR0402720

[ALP17] A. Ananyevskiy, M. Levine, and I. Panin, Witt sheaves and the $\eta$-inverted sphere spectrum, J. Topol. 10 (2017), no. 2, 370-385, DOI 10.1112/topo.12015. MR3653315

[ARØ17] A. Ananyevskiy, O. Röndigs, and P. A. Østvær, On very effective hermitian $K$-theory (2017), preprint, available at arXiv:1712.01349

[And18] M. J. Andrews, New families in the homotopy of the motivic sphere spectrum, Proc. Amer. Math. Soc. 146 (2018), no. 6, 2711-2722, DOI 10.1090/proc/13940. MR3778171

[AM17] M. Andrews and H. Miller, Inverting the Hopf map, J. Topol. 10 (2017), no. 4, 11451168, DOI 10.1112/topo.12034. MR3743072

[AF15] A. Asok and J. Fasel, Splitting vector bundles outside the stable range and $\mathbb{A}^{1}$ homotopy sheaves of punctured affine spaces, J. Amer. Math. Soc. 28 (2015), no. 4, 1031-1062, DOI 10.1090/S0894-0347-2014-00818-3. MR3369908

[AWW17] A. Asok, K. Wickelgren, and B. Williams, The simplicial suspension sequence in $\mathbb{A}^{1}$ homotopy, Geom. Topol. 21 (2017), no. 4, 2093-2160, DOI 10.2140/gt.2017.21.2093. MR3654105

[AH61] M. F. Atiyah and F. Hirzebruch, Vector bundles and homogeneous spaces, Proc. Sympos. Pure Math., Vol. III, American Mathematical Society, Providence, R.I., 1961, pp. 7-38. MR0139181

[Bac18] T. Bachmann, Motivic and real étale stable homotopy theory, Compos. Math. 154 (2018), no. 5, 883-917, DOI 10.1112/S0010437X17007710. MR3781990

[BMT70] M. G. Barratt, M. E. Mahowald, and M. C. Tangora, Some differentials in the Adams spectral sequence. II, Topology 9 (1970), 309-316. MR0266215 (42 \#1122)

[Beh07] M. Behrens, Some root invariants at the prime 2, Proceedings of the Nishida Fest (Kinosaki 2003), Geom. Topol. Monogr., vol. 10, Geom. Topol. Publ., Coventry, 2007, pp. 1-40, DOI 10.2140/gtm.2007.10.1. MR2402775

[BI] E. Belmont and D. C. Isaksen, The $\mathbb{R}$-motivic Adams spectral sequence, in preparation.

[Boa99] J. M. Boardman, Conditionally convergent spectral sequences, Homotopy invariant algebraic structures (Baltimore, MD, 1998), Contemp. Math., vol. 239, Amer. Math. Soc., Providence, RI, 1999, pp. 49-84, DOI 10.1090/conm/239/03597. MR1718076

[Bru84] R. Bruner, A new differential in the Adams spectral sequence, Topology 23 (1984), no. 3, 271-276, DOI 10.1016/0040-9383(84)90010-7. MR770563 (86c:55016) 
[Bru89] R. R. Bruner, Calculation of large Ext modules, Computers in geometry and topology (Chicago, IL, 1986), Lecture Notes in Pure and Appl. Math., vol. 114, Dekker, New York, 1989, pp. 79-104. MR988692

[Bru93] - Ext in the nineties, Algebraic topology (Oaxtepec, 1991), Contemp. Math., vol. 146, Amer. Math. Soc., Providence, RI, 1993, pp. 71-90. MR1224908

[Bru97] - The cohomology of the mod 2 Steenrod algebra: A computer calculation 37 (1997).

[BG95] R. Bruner and J. Greenlees, The Bredon-Löffler conjecture, Experiment. Math. 4 (1995), no. 4, 289-297. MR1387694

[DFHH14] C. L. Douglas, J. Francis, A. G. Henriques, and M. A. Hill (eds.), Topological modular forms, Mathematical Surveys and Monographs, vol. 201, American Mathematical Society, Providence, RI, 2014. MR3223024

[DK18] A. Druzhinin and J. I. Kylling, Framed correspondences and the zeroth stable motivic homotopy group in odd characteristic (2018), preprint, available at arXiv:1809.03238.

[Dug14] D. Dugger, Coherence for invertible objects and multigraded homotopy rings, Algebr. Geom. Topol. 14 (2014), no. 2, 1055-1106, DOI 10.2140/agt.2014.14.1055. MR3180827

[DI10] D. Dugger and D. C. Isaksen, The motivic Adams spectral sequence, Geom. Topol. 14 (2010), no. 2, 967-1014, DOI 10.2140/gt.2010.14.967. MR2629898 (2011e:55024)

[DI13] _ Motivic Hopf elements and relations, New York J. Math. 19 (2013), 823-871. MR3141814

[DI17] — Low-dimensional Milnor-Witt stems over $\mathbb{R}$, Ann. K-Theory 2 (2017), no. 2, 175-210. MR3590344

[ELSØ17] E. Elmanto, M. Levine, M. Spitzweck, and P. A. Østvær, Motivic Landweber exact theories and étale cohomology (2017), preprint, available at arXiv:1711.06258.

[Ghe17] B. Gheorghe, Exotic motivic periodicities (2017), preprint, available at arXiv:1709.00915.

[Ghe] The motivic cofiber of $\tau$, Doc. Math., to appear.

[GWX18] B. Gheorghe, G. Wang, and Z. Xu, The special fiber of the motivic deformation of the stable homotopy category is algebraic (2018), preprint, available at arXiv:1809.09290.

[GI15] B. J. Guillou and D. C. Isaksen, The $\eta$-local motivic sphere, J. Pure Appl. Algebra 219 (2015), no. 10, 4728-4756, DOI 10.1016/j.jpaa.2015.03.004. MR3346515

[GI16] , The $\eta$-inverted $\mathbb{R}$-motivic sphere, Algebr. Geom. Topol. 16 (2016), no. 5, 3005-3027. MR3572357

[GRSØ12] J. J. Gutiérrez, O. Röndigs, M. Spitzweck, and P. A. Østvær, Motivic slices and coloured operads, J. Topol. 5 (2012), no. 3, 727-755, DOI 10.1112/jtopol/jts015. MR2971612

[Hil11] M. A. Hill, Ext and the motivic Steenrod algebra over $\mathbb{R}$, J. Pure Appl. Algebra 215 (2011), no. 5, 715-727, DOI 10.1016/j.jpaa.2010.06.017. MR2747214 (2012i:55020)

[HHR16] M. A. Hill, M. J. Hopkins, and D. C. Ravenel, On the nonexistence of elements of Kervaire invariant one, Ann. of Math. (2) 184 (2016), no. 1, 1-262, DOI 10.4007/annals.2016.184.1.1. MR3505179

[Hor05] J. Hornbostel, $A^{1}$-representability of Hermitian K-theory and Witt groups, Topology 44 (2005), no. 3, 661-687, DOI 10.1016/j.top.2004.10.004. MR2122220

[Hor18] _ Some comments on motivic nilpotence, Trans. Amer. Math. Soc. 370 (2018), no. 4, 3001-3015, DOI 10.1090/tran/7324. With an appendix by Marcus Zibrowius. MR3748592

[Hoy15] M. Hoyois, From algebraic cobordism to motivic cohomology, J. Reine Angew. Math. 702 (2015), 173-226, DOI 10.1515/crelle-2013-0038. MR3341470

[HKØ17] M. Hoyois, S. Kelly, and P. A. Østvær, The motivic Steenrod algebra in positive characteristic, J. Eur. Math. Soc. (JEMS) 19 (2017), no. 12, 3813-3849, DOI 10.4171/JEMS/754. MR3730515

[HK01] P. Hu and I. Kriz, Some remarks on Real and algebraic cobordism, K-Theory 22 (2001), no. 4, 335-366, DOI 10.1023/A:1011196901303. MR1847399

[HKO11a] P. Hu, I. Kriz, and K. Ormsby, Remarks on motivic homotopy theory over algebraically closed fields, J. K-Theory 7 (2011), no. 1, 55-89, DOI 10.1017/is010001012jkt098. MR2774158 (2012b:14040)

[HKO11b] _ Convergence of the motivic Adams spectral sequence, J. K-Theory 7 (2011), no. 3, 573-596, DOI 10.1017/is011003012jkt150. MR2811716 
[Isa] D. C. Isaksen, Stable stems, Mem. Amer. Math. Soc., to appear.

[Isa14] _ Classical and motivic Adams charts (2014), preprint, available at arXiv: 1401.4983

[IS11] D. C. Isaksen and A. Shkembi, Motivic connective K-theories and the cohomology of A(1), J. K-Theory 7 (2011), no. 3, 619-661, DOI 10.1017/is011004009jkt154. MR2811718 (2012h:19012)

[IWX] D. C. Isaksen, G. Wang, and Z. Xu, More stable stems, in preparation.

[IX15] D. C. Isaksen and Z. Xu, Motivic stable homotopy and the stable 51 and 52 stems, Topology Appl. 190 (2015), 31-34, DOI 10.1016/j.topol.2015.04.008. MR3349503

[Jar00] J. F. Jardine, Motivic symmetric spectra, Doc. Math. 5 (2000), 445-552. MR1787949

[Joa15] R. Joachimi, Thick ideals in equivariant and motivic stable homotopy categories (2015), Ph.D. thesis.

[JW73] D. C. Johnson and W. S. Wilson, Projective dimension and Brown-Peterson homology, Topology 12 (1973), 327-353, DOI 10.1016/0040-9383(73)90027-X. MR0334257

[Kra18] A. Krause, Periodicity in motivic homotopy theory and over $B P_{*} B P(2018)$, Ph.D. thesis.

[KW18] J. I. Kylling and G. M. Wilson, Strong convergence in the motivic Adams spectral sequence (2018), preprint.

[Lev08] M. Levine, The homotopy coniveau tower, J. Topol. 1 (2008), no. 1, 217-267, DOI $10.1112 / \mathrm{jtopol} / \mathrm{jtm} 004$. MR2365658

[Lev14] _ A comparison of motivic and classical stable homotopy theories, J. Topol. 7 (2014), no. 2, 327-362, DOI 10.1112/jtopol/jtt031. MR3217623

[Mag02] B. A. Magurn, An algebraic introduction to K-theory, Encyclopedia of Mathematics and its Applications, vol. 87, Cambridge University Press, Cambridge, 2002. MR1906572

[MT67] M. Mahowald and M. Tangora, Some differentials in the Adams spectral sequence, Topology 6 (1967), 349-369. MR0214072 (35 \#4924)

[Man18] L. Mantovani, Localizations and completions in motivic homotopy theory (2018), preprint, available at arXiv:1810.04134

[May65] J. P. May, The cohomology of restricted Lie algebras and of Hopf algebras, Bull. Amer. Math. Soc. 71 (1965), 372-377, DOI 10.1090/S0002-9904-1965-11300-3. MR0185595

[13] Algebraic K-theory and motivic cohomology, Oberwolfach Rep. 10 (2013), no. 2, 1861-1913, DOI 10.4171/OWR/2013/32. Abstracts from the workshop held June 2329, 2013; Organized by T. Geisser, A. Huber-Klawitter, U. Jannsen and M. Levine. MR3013936

[Mil75] H. R. Miller, Some algebraic aspects of the Adams-Novikov spectral sequence, ProQuest LLC, Ann Arbor, MI, 1975. Thesis (Ph.D.)-Princeton University. MR2625232

[Mil60] J. Milnor, On the cobordism ring $\Omega^{*}$ and a complex analogue. I, Amer. J. Math. 82 (1960), 505-521, DOI 10.2307/2372970. MR0119209

[Mil69] _ Algebraic K-theory and quadratic forms, Invent. Math. 9 (1969/1970), 318344. MR0260844

[Mor99a] F. Morel, Suite spectrale d'Adams et invariants cohomologiques des formes quadratiques, C. R. Acad. Sci. Paris Sér. I Math. 328 (1999), no. 11, 963-968, DOI 10.1016/S0764-4442(99)80306-1 (French, with English and French summaries). MR1696188 (2000d:11056)

[Mor99b] — Théorie homotopique des schémas, Astérisque 256 (1999), vi+119 (French, with English and French summaries). MR1693330 (2000j:14031)

[Mor04a] _ An introduction to $\mathbb{A}^{1}$-homotopy theory, Contemporary developments in algebraic $K$-theory, ICTP Lect. Notes, XV, Abdus Salam Int. Cent. Theoret. Phys., Trieste, 2004, pp. 357-441 (electronic). MR2175638 (2006m:19007)

[Mor04b] _ On the motivic $\pi_{0}$ of the sphere spectrum, Axiomatic, enriched and motivic homotopy theory, NATO Sci. Ser. II Math. Phys. Chem., vol. 131, Kluwer Acad. Publ., Dordrecht, 2004, pp. 219-260, DOI 10.1007/978-94-007-0948-5_7. MR2061856

[Mor06] _ Rationalized motivic sphere spectrum and rational motivic cohomology (2006), preprint.

[Mor12] _ , $\mathbb{A}^{1}$-algebraic topology over a field, Lecture Notes in Mathematics, vol. 2052, Springer, Heidelberg, 2012. MR2934577 
[MV99] F. Morel and V. Voevodsky, $\mathbf{A}^{1}$-homotopy theory of schemes, Inst. Hautes Études Sci. Publ. Math. 90 (1999), 45-143 (2001). MR1813224 (2002f:14029)

[Mos70] R. M. F. Moss, Secondary compositions and the Adams spectral sequence, Math. Z. 115 (1970), 283-310. MR0266216 (42 \#1123)

[Nas] C. Nassau, http://www.nullhomotopie.de/

[NSØ09] N. Naumann, M. Spitzweck, and P. A. Østvær, Motivic Landweber exactness, Doc. Math. 14 (2009), 551-593. MR2565902

[NSØ15] — Existence and uniqueness of $E_{\infty}$ structures on motivic $K$-theory spectra, J. Homotopy Relat. Struct. 10 (2015), no. 3, 333-346, DOI 10.1007/s40062-013-0062-3. MR3385689

[Nes18] A. Neshitov, Framed correspondences and the Milnor-Witt K-theory, J. Inst. Math. Jussieu 17 (2018), no. 4, 823-852, DOI 10.1017/S1474748016000190. MR3835524

[Nov67] S. P. Novikov, Methods of algebraic topology from the point of view of cobordism theory, Izv. Akad. Nauk SSSR Ser. Mat. 31 (1967), 855-951 (Russian). MR0221509

[OVV07] D. Orlov, A. Vishik, and V. Voevodsky, An exact sequence for $K_{*}^{M} / 2$ with applications to quadratic forms, Ann. of Math. (2) 165 (2007), no. 1, 1-13, DOI 10.4007/annals.2007.165.1. MR2276765

[Orm11] K. M. Ormsby, Motivic invariants of p-adic fields, J. K-Theory 7 (2011), no. 3, 597618, DOI 10.1017/is011004017jkt153. MR2811717

[OØ13] K. M. Ormsby and P. A. Østvær, Motivic Brown-Peterson invariants of the rationals, Geom. Topol. 17 (2013), no. 3, 1671-1706, DOI 10.2140/gt.2013.17.1671. MR3073932

[OØ14] Stable motivic $\pi_{1}$ of low-dimensional fields, Adv. Math. 265 (2014), 97-131, DOI 10.1016/j.aim.2014.07.024. MR3255457

[Pst18] P. Pstragowski, Synthetic spectra and the cellular motivic category (2018), preprint, available at arXiv:1803.01804

[Rav86] D. C. Ravenel, Complex cobordism and stable homotopy groups of spheres, Pure and Applied Mathematics, vol. 121, Academic Press, Inc., Orlando, FL, 1986. MR860042 (87j:55003)

[Rön] O. Röndigs, On the $\eta$-inverted sphere, Tata Institute of Fundamental Research Publications 19 , to appear.

[RØ08] O. Röndigs and P. A. Østvær, Modules over motivic cohomology, Adv. Math. 219 (2008), no. 2, 689-727, DOI 10.1016/j.aim.2008.05.013. MR2435654

[RØ16] O. Röndigs and P. A. Østvær, Slices of hermitian K-theory and Milnor's conjecture on quadratic forms, Geom. Topol. 20 (2016), no. 2, 1157-1212, DOI 10.2140/gt.2016.20.1157. MR3493102

[RSØ18] O. Röndigs, M. Spitzweck, and P. A. Østvær, The motivic Hopf map solves the homotopy limit problem for K-theory, Doc. Math. 23 (2018), 1405-1424.

[RSØa] — The first stable homotopy groups of motivic spheres, Ann. of Math., to appear.

[RSØb] - The second stable homotopy groups of motivic spheres, in preparation.

[Ser02] J.-P. Serre, Galois cohomology, Corrected reprint of the 1997 English edition, Springer Monographs in Mathematics, Springer-Verlag, Berlin, 2002. Translated from the French by Patrick Ion and revised by the author. MR1867431

[Sou79] C. Soulé, $K$-théorie des anneaux d'entiers de corps de nombres et cohomologie étale, Invent. Math. 55 (1979), no. 3, 251-295, DOI 10.1007/BF01406843 (French). MR553999

[Spi10] M. Spitzweck, Relations between slices and quotients of the algebraic cobordism spectrum, Homology Homotopy Appl. 12 (2010), no. 2, 335-351. MR2771593

[Spi12] — A commutative $\mathbb{P}^{1}$-spectrum representing motivic cohomology over Dedekind domains (2012), preprint, available at arXiv:1207.4078

[Spi14] — Algebraic cobordism in mixed characteristic (2014), preprint, available at arXiv: 1404.2542

[SØ12] M. Spitzweck and P. A. Østvær, Motivic twisted K-theory, Algebr. Geom. Topol. 12 (2012), no. 1, 565-599, DOI 10.2140/agt.2012.12.565. MR2916287

[Sta16] S.-T. Stahn, The motivic Adams-Novikov spectral sequence at odd primes over $\mathbb{C}$ and $\mathbb{R}$ (2016), preprint, available at arXiv:1606.06085.

[Tho85] R. W. Thomason, Algebraic K-theory and étale cohomology, Ann. Sci. École Norm. Sup. (4) 18 (1985), no. 3, 437-552. MR826102 
[Tho16] R. Thornton, The homogeneous spectrum of Milnor-Witt K-theory, J. Algebra 459 (2016), 376-388, DOI 10.1016/j.jalgebra.2016.04.012. MR3503978

[Vez01] G. Vezzosi, Brown-Peterson spectra in stable $\mathbb{A}^{1}$-homotopy theory, Rend. Sem. Mat. Univ. Padova 106 (2001), 47-64. MR1876212

[Voe98] V. Voevodsky, $\mathbb{A}^{1}$-homotopy theory, Proceedings of the International Congress of Mathematicians, Vol. I (Berlin, 1998), 1998, pp. 579-604 (electronic). MR1648048 (99j:14018)

[Voe02a] - Open problems in the motivic stable homotopy theory. I, Motives, polylogarithms and Hodge theory, Part I (Irvine, CA, 1998), Int. Press Lect. Ser., vol. 3, Int. Press, Somerville, MA, 2002, pp. 3-34. MR1977582

[Voe02b] — A possible new approach to the motivic spectral sequence for algebraic $K$-theory, Recent progress in homotopy theory (Baltimore, MD, 2000), Contemp. Math., vol. 293, Amer. Math. Soc., Providence, RI, 2002, pp. 371-379, DOI 10.1090/conm/293/04956. MR1890744

[Voe03a] _ Reduced power operations in motivic cohomology, Publ. Math. Inst. Hautes Études Sci. 98 (2003), 1-57, DOI 10.1007/s10240-003-0009-z. MR2031198 (2005b:14038a)

[Voe03b] Motivic cohomology with Z/2-coefficients, Publ. Math. Inst. Hautes Études Sci. 98 (2003), 59-104, DOI 10.1007/s10240-003-0010-6. MR2031199 (2005b:14038b)

[Voe11] no. 1, 401-438. MR2811603

[WX] G. Wang and Z. Xu, On the uniqueness of the smooth structure of the 61-sphere, Ann. of Math., to appear.

[Wei13] C. A. Weibel, The $K$-book, Graduate Studies in Mathematics, vol. 145, American Mathematical Society, Providence, RI, 2013. An introduction to algebraic $K$-theory. MR3076731

[Wil18] G. M. Wilson, The eta-inverted sphere over the rationals, Algebr. Geom. Topol. 18 (2018), no. 3, 1857-1881, DOI 10.2140/agt.2018.18.1857. MR3784021

[WØ17] G. M. Wilson and P. A. Østvær, Two-complete stable motivic stems over finite fields, Algebr. Geom. Topol. 17 (2017), no. 2, 1059-1104. MR3623682

Department of Mathematics, Wayne State University, Detroit, Mi 48202, USA

E-mail address: isaksen@wayne.edu

Department of Mathematics, University of Oslo, 0316 Oslo, Norway

E-mail address: paularne@math.uio.no 